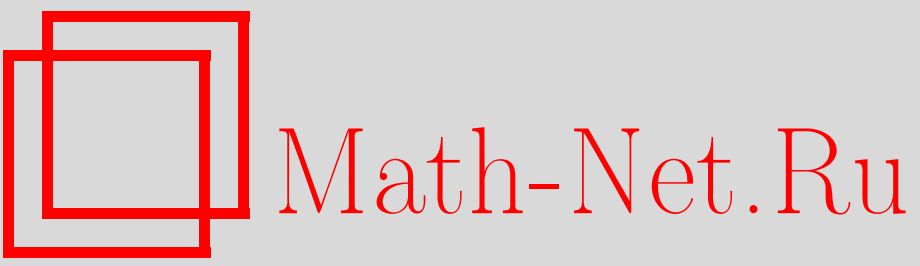

Д. А. Лейтес, И. М. Щепочкина, Как квантовать антискобку?, ТМФ, 2001, том 126, номер 3, 339-369

DOI: https://doi.org/10.4213/tmf435

Использование Общероссийского математического портала Math-Net.Ru подразумевает, что вы прочитали и согласны с пользовательским соглашением

http://www.mathnet.ru/rus/agreement

Параметры загрузки:

IP : 3.85 .183 .62

26 апреля 2023 г., 15:41:37 


\author{
ТЕОРЕТИЧЕСКАЯ \\ И МАТЕМАТИЧЕСКАЯ \\ ФИЗИКА \\ Том 126, № 3 \\ март, 2001
}

(C) 2001 г

Д. А. Лейтес* , И.М. Шепочкина ${ }^{\dagger}$

\title{
КАК КВАНТОВАТЬ АНТИСКОБКУ?
}

Доказано, что, в отличие от алгебры po $(2 n \mid m)$, ее фактор по центру - супералгебра Ли $\mathfrak{h}(2 n \mid m)$ гамильтоновых векторных полей с полиномиальными коэффициентами имеет исключительные дополнительные деформации при $(2 n \mid m)=(2 \mid 2)$ и только в этой суперразмерности. Этот результат связывается с полным описанием квантований (и деформаций) антискобки (называемой также скобкой Схоутена или Бюттен). Оказывается, что пространство, в котором действует деформированная алгебра Ли (результат квантования алгебры Пуассона), совпадает с простейшим пространством, в котором действует алгебра Ли коммутационных соотношений. Это совпадение не обязательно в случае супералгебр Ли.

\section{1. ВВЕДЕНИЕ}

Задача, которую мы рассматриваем, часто формулируется недостаточно четко. Чтобы избежать недоразумений и сформулировать наш основной результат, мы постараемся описать ее как можно более аккуратно. Утомительные вычисления, включая изложение важной, но труднодоступной работы [1], будут помешены в сети Интернет по адресу xxx.lanl.gov, однако все необходимые предварительные сведения представлены в приложении.

В 1977 году, когда один из авторов (Д. Лейтес) опубликовал статью [2], М. Маринов спросил его: "Как квантовать эту "новую механику"? Неужели с нечетной постоянной Планка?" В 1987 году Стернберг повторил этот вопрос в связи со своей работой [3]. Предварительный ответ можно найти в работе [4], где выявлена важность нечетных параметров и "странного" супераналога $\mathfrak{q}(n)$ алгебры Ли $\mathfrak{g l}(n)$. В данной статье мы, хотя и уделяем основное внимание несколько иному вопросу, все же, как и в [4], не устаем подчеркивать важность языка точек и нечетных параметров для супералгебр Ли, особенно когда речь идет об их деформациях.

1.1. Общая постановка задачи. Существует много различных интерпретаций понятия "квантование". Мы рассматриваем квантование как деформацию, а для этого совершенно необходимо начать с ясного описания того класса, внутри которого мы деформируем наш объект. Например, в простейшем случае, когда супермногообразие $\mathcal{M}$

${ }^{*}$ Department of Mathematics, University of Stockholm, Stockholm, Sweden. E-mail: mleites@matematik.su.se

${ }^{\dagger}$ Независимый московский университет, Москва, Россия. E-mail: ira@paramonova.mccme.ru 
есть $\mathbb{C}^{2 n \mid m}$ (или $\mathbb{R}^{2 n \mid m}$ ), наделенное симплектической структурой, рассмотрим суперпространство $\mathcal{F}$ функций на $\mathcal{M}$. Сушествуют две естественные структуры на $\mathcal{F}$ : структура ассоциативной (и суперкоммутативной) супералгебры и структура супералгебры Ли, называемой супералгеброй Пуассона и обозначаемой po $(2 n \mid m)$. Поэтому прежде всего мы должны выбрать одну из двух задач:

1) описание деформации (не обязательно суперкоммутативной) ассоциативнойсупералгебры $\mathcal{F}$

или

2) описание деформации супералгебры Лu po $(2 n \mid m)$.

Обе задачи можно решить, вычисляя некоторые когомологии (когомологии Хохшильда для ассоциативных алгебр или лиевы когомологии для алгебр Ли; переход к супералгебрам осуществляется с помощью правила знаков). Задача 1 рассматривалась под разными углами Флато, Лихнеровичем, Нирославским и Власовым, Леконтом и де Уилдом, Дринфельдом, Федосовым, Концевичем и др. (см., например, [5], [6]). Всегда было ясно, что задачи 1 и 2 связаны друг с другом; в настоящей статье мы настаиваем на том, чтобы не подменять одну из них другой, а отчетливо разделить их и сосредоточиться на задаче 2. Дирак был, вероятно, первым, кто ей занимался; долгое время единственной работой, в которой изучалась деформация лиевской структуры, была статья Вея [7] (ср. [8]); в [4] и в настояшей работе мы продолжаем эти исследования. Совершенно иной подход к квантованию принадлежит Березину [9], который считал, что размерности деформируемых алгебр могут меняться, и исследовал сходимость формальных деформаций.

Что касается задачи 1, то Шерешевский [10] был, по-видимому, первым, кто показал, что пространство деформаций ассоциативных структур на $\mathcal{F}(\mathcal{M})$ не меньше, чем пространство аффинных связностей на $\mathcal{M}$, и, следовательно, слишком обширно, чтобы представлять какой-либо интерес. Концевич [11] понял, что это пространство следует рассматривать по модулю некоторых калибровочных преобразований, которые делают фактор-пространство обозримым (точнее, одномерным). Суперизация этого результата - стандартная процедура, проделанная в работе [12].

До появления работы Концевича, для того чтобы уменьшить число деформаций $a c$ социативной структуры, обычно предполагали, что производная деформации пропорциональна заданной лиевской структуре, т.е. фактически заменяли задачу 1 задачей 2. Это ясно из сравнения работ [11] и [9].

ЗАмЕчАНИЕ. Отметим, что Концевич рассматривал также скобку Ли, построенную по любому нечетному бивекторному полю, не обязательно невырожденному. Такие скобки иногда также называются скобками Пуассона, что приводит к дополнительным недоразумениям.

1.2. Когомологии зависят от класса функций. Число неэквивалентных деформаций (супер)алгебры Ли g может зависеть и от типа функций, входящих в определение $\mathfrak{g}$ (гладких, аналитических, полиномиальных и т.д.): это видно из сравнения параметрического семейства $\mathfrak{s v e c t}_{\lambda}^{L}(1 \mid n)$ бездивергентных векторных полей, коэффициентами которых являются полиномы Лорана [13], с недеформируемой супералгеброй Ли $\mathfrak{s v e c t}(1 \mid n)$ бездивергентных векторных полей с полиномиальными коэффициентами. 
Единственность деформации $\mathfrak{p o}(2 n)$ считается давно и хорошо известным фактом. Когда Баталин и Тютин [14] действительно доказали единственность деформации пуассоновых супералгебр Ли ро $(2 n \mid m)$ (порожденных функциями определенного класса), им было трудно опубликовать этот результат, поскольку он воспринимался как "известный”, хотя до этого не было опубликовано доказательства даже для четного случая (см. [7], обзор [15], где обсуждается сушествование деформации, и сборник работ на эту тему [8]). Однако здесь следует быть очень аккуратным, так как для произвольных функций утверждение неверно, его справедливость зависит от класса функций: для полиномов единственность имеет место, а для полиномов Лорана нет (см. [16]).

1.3. Что такое супералгебра Ли? Понятие супералгебр Ли было введено в топологии в 1930-х годах или даже раньше. Когда кто-то пытается предложить "лучшее" определение для понятия, введенного более 70 лет назад и, вроде бы, устоявшегося, это может показаться по меньшей мере странным. Тем не менее ответ на вопрос, что же такое супералгебра Ли, до сих пор не является общеизвестным. В самом деле, наивное определение (“применяйте правило знаков к определению алгебры Ли”) явно недостаточно для исследования (сингулярных) супермногообразий деформаций и для приложений теории представлений в математической физике, например при изучении коприсоединенного представления супергруппы Ли: супергруппа может действовать на супермногообразиях, но не на каком бы то ни было суперпространстве, так как это объекты разных категорий. Для того чтобы деформировать супералгебры Ли или применять теоретико-групповой подход в суперслучае, нужно уметь строить супермногообразие по суперпространству и наоборот.

Надлежащее определение супералгебры Ли дано в работе [4].

ОПреДЕЛЕниЕ. Пусть $L=L_{\overline{0}} \oplus L_{\overline{1}}-$ "наивная" супералгебра Ли. Ей отвечает супералљебра Ли в категории супермногообразий - линейное супермногообразие $\mathcal{L}=$ $\left(L_{\overline{0}}, \mathcal{O}\right)$, где пучок функций $\mathcal{O}$ состоит из функций на $L_{\overline{0}}$ со значениями в грассмановой алгебре от $L_{\overline{1}}^{*}$; это супермногообразие должно быть таким, что для “любой” (скажем, конечно-порожденной или из какой-нибудь иной подходящей категории) суперкоммутативной супералгебры $C$ пространство $\mathcal{L}(C)=\operatorname{Hom}(\operatorname{Spec} C, \mathcal{L})$, называемое пространством $C$-точек из $\mathcal{L}$, является алгеброй Ли и соответствие $C \rightarrow \mathcal{L}(C)$ является функтором в $C$.

Этот подход был введен в алгебраическую геометрию Вейлем в 1954 году, в теории супералгебр он называется языком точек или семейств [17]. Приведенное выше определение может показаться чересчур сложным, но, к счастью, можно показать, что соответствие $\mathcal{L} \longleftrightarrow L$ является взаимно однозначным, и алгебра Ли $\mathcal{L}(C)$, также обозначаемая $L(C)$, допускает очень простое описание: $L(C)=(L \otimes C)_{\overline{0}}$.

Гомоморфизм $\rho: L_{1} \rightarrow L_{2}$ супералгебр Ли в этих терминах - это функторный морфизм, т.е. семейство гомоморфизмов алгебры Ли $\rho_{C}: L_{1}(C) \rightarrow L_{2}(C)$, согласованных с морфизмами суперкоммутативных супералгебр $C \rightarrow C^{\prime}$. В частности, представление супералгебры Ли $L$ в суперпространстве $V$ - это гомоморфизм $\rho: L \rightarrow \mathfrak{g l}(V)$, т.е. семейство гомоморфизмов $\rho_{C}: L(C) \rightarrow(\mathfrak{g l}(V) \otimes C)_{\overline{0}}$ алгебр Ли.

ПримеР. Рассмотрим представление $\rho: \mathfrak{g} \rightarrow \mathfrak{g l}(V)$. Касательное пространство суперпространства деформаций представления $\rho$ в точке, отвечаюшей представлению $\rho$, 
изоморфно $H^{1}\left(\mathfrak{g} ; V \otimes V^{*}\right)$. Например, если $\mathfrak{g}$ есть $(0 \mid n)$-мерная (т.е. чисто нечетная) супералгебра Ли (с единственно возможной скобкой, тождественно равной нулю), то ее неприводимые представления - это только тривиальное представление $\mathbf{1}$ и П(1). Ясно, что $\mathbf{1} \otimes \mathbf{1}^{*} \simeq \Pi(\mathbf{1}) \otimes \Pi(\mathbf{1})^{*} \simeq \mathbf{1}$, и, поскольку $\mathfrak{g}$ коммутативна, дифференциал в коцепном комплексе тривиален. Таким образом, $H^{2}(\mathfrak{g} ; \mathbf{1})=E^{1}(\mathfrak{g})=\mathfrak{g}^{*}$; значит, существует $\operatorname{dim} \mathfrak{g}$ нечетных параметров инфинитезимальных деформаций тривиального представления. Если бы мы рассматривали $\mathfrak{g}$ "наивно", то потеряли бы все нечетные параметры.

Какие из этих инфинитезимальных деформаций продолжаются до глобальных, вопрос отдельный и более сложный, обычно решаемый ad hoc (индивидуально) (см. [18]).

В настояшей работе мы сталкиваемся с похожей ситуацией: мы деформируем структуру супералгебры Ли, т.е. суперскобку. Деформации любой суперструктуры могут, конечно же, иметь нечетные параметры. Манин [19] пишет, что это очевидно, но нам представляется, что он слишком оптимистичен: даже классики иногда все еще не рассматривают нечетные параметры (см., например, [20]). Физики легче принимают нечетные (и другие инфинитезимальные) параметры: нечетные параметры являются краеугольным камнем суперсимметрии Весса и Зумино [21]; Виттен в серии замечательных работ демонстрирует важность нечетных параметров (см., например, [22]). Работы Виттена вызвали к жизни лавину исследований, среди которых мы хотели бы выделить [19], [23]-[25].

1.4. Квантование, как мы его понимаем. Квантование супералгебры Ли $\mathfrak{p o}(2 n \mid m)$ распадается на два этапа:

1) деформация супералгебры Ли po $(2 n \mid m)$;

2) реализация деформированной супералгебры Ли операторами в некотором (фоковском) пространстве.

Существует также этап предквантования, т.е. реализации $\mathfrak{p o}(2 n \mid m)$ в некоторой "классической версии" фоковского пространства.

Реализация этапов 1 и 2 представляется рутинным занятием, их суперизация содержит только два новых момента. Первый момент состоит в том, что супералгебра $\mathfrak{p o}(2 n \mid m)$ для нечетного $m$ реализуется не всеми дифференциальными операторами, а лишь частью, сохраняюшей комплексную структуру, заданную нечетным оператором, и аналогичной "странному” аналогу общей линейной алгебры Ли (подробнее см. в [4]). Второй новый момент описан в п. 1.5.

Полное описание предквантований можно найти в работах [26]-[28]. С предквантованием связано также описание представления соотношений (анти)коммутации. Оказывается, что пространство, в котором действует деформированная алгебра Ли (результат квантования алгебры po $(2 n \mid 0))$, совпадает с простейшим пространством, в котором действует представление соотношений (анти)коммутации (подход, отличный от нашего, см. в [29]). Это совпадение не обязательно в случае супералгебр Ли.

В настоящей статье мы рассматриваем нечетный аналог скобки Пуассона, т.е. антискобку, именуемую в зависимости от контекста также скобкой Схоутена или Бюттен, и деформации антискобки, отличные от квантований. Для каждой из супералгебр Ли с такими скобками, т.е. для алгебр $\mathfrak{p o}(2 n \mid m), \mathfrak{b}(n)$ и для каждого элемента $\mathfrak{b}_{\lambda}(n)$ из однопараметрического семейства деформаций супералгебры Ли $\mathfrak{b}(n)$, мы исследуем ее 
квантования в вышеприведенном смысле.

Говоря об антискобке, напомним, что супералгебра Бюттен $\mathfrak{b}(n)$ - это суперпространство функций на $(n \mid n)$-мерном суперпространстве с обрашенной четностью и структурой супералгебры Ли, задаваемой антискобкой; $\mathfrak{b}(n)$ может трактоваться также как суперпространство векторных полей с обрашенной четностью на $(p \mid q)$-мерном суперпространстве при любых $p$ и $q$ таких, что $p+q=n ; p, q \geqslant 0$, и структурой супералгебры Ли, задаваемой скобкой Схоутена.

ЗАмЕчаниЕ. Операция, которую мы называем скобкой Бюттен, была открыта Схоутеном еше до развития теории супералгебр Ли; Бюттен первой доказала, что эта операция задает структуру супералгебры Ли. Интерпретация супералгебры Бюттен как аналога алгебры Пуассона, а элементов супералгебры $\mathfrak{l}(n)=\mathfrak{b}(n) /$ const как аналогов гамильтоновых векторных полей обнаружена в [2]. Скобка Бюттен и "нечетная механика", введенные в [2], были переоткрыты Баталиным и Вилковыским; под названием "антискобка" она стала расхожим понятием, важность которого показана в серии работ Баталина и Вилковыского, а затем и многих других (см. обзоры [30], [31]).

Не каждая деформация может рассматриваться как квантование. Грубо говоря, если в качестве исходной мы берем (супер)алгебру Ли векторных полей на суперпространстве определенной размерности, то после квантования мы должны получить алгебру, обладаюшую представлением в суперпространстве половинной функциональной размерности. Деформации $\mathfrak{b}(n)$, определенные в (2.1) и (2.2) (см. ниже), обозначаются $\mathfrak{b}_{\lambda}(n)$ (см. приложение). Они не являются результатом квантования супералгебры $\mathfrak{b}(n)$ в вьшеуказанном смысле (у этой супералгебры Ли нет представлений половинной функциональной размерности). То, что мы называем квантованием, просто записывается похожим на единственное квантование алгебры Пуассона образом.

Нечетная версия предквантований, т.е. представлений $\mathfrak{b}_{\lambda}(n)$, и связанное с этим описание представлений $\mathfrak{l}(n)$ приведены в работах [32], [33].

Этап 1 описан Кочетковым [1], [34]-[37] для супералгебры Бюттен $\mathfrak{b}_{\lambda}(n)$ и для $\mathfrak{h}(2 n \mid m)$, за исключением случая $n m \neq 0$. В настоящей статье мы исправляем результат Кочеткова и завершаем этап 2, начатый в работе [4].

1.5. Несколько фоковских пространств для соотношений (анти)коммутации. Мы рассматриваем ро и ее "нечетный" аналог $\mathfrak{b}$, так же как и деформации последней $\mathfrak{b}_{\lambda}$, как супералгебры Ли. Эти супералгебры Ли, и особенно их квантовые аналоги, в каком-то смысле являются аналогами $\mathfrak{g l}(V)$. Алгебра Ли $\mathfrak{g l}(V)$ имеет много неприводимых представлений (реализуюшихся в тензорах от $V$ и $V^{*}$, в модулях со старшим весом и т.д.). Это же можно сказать и обо всех вышеупомянутых супералгебрах Ли.

Напротив, ассоциативная алгебра $\operatorname{Mat}(V)$ эндоморфизмов $V$, или в матричной форме $\operatorname{Mat}(\operatorname{dim} V)$, хотя и изоморфна $\mathfrak{g l}(V)$, как векторное пространство, но обладает лиш одним неприводимым модулем $V$ (так же как и супервариант алгебры $\operatorname{Mat}(V)$ и даже “странная" версия $Q(V))$. Этот модуль $V$ и называется фоковским пространством. Таким образом, фоковское пространство является аналогом стандартного или тождественного представления для $\mathfrak{g l}(V)$. Это означает, что когда мы рассматриваем (супер)алгебры Ли, нам следует выбирать в качестве аналогов фоковского пространства "минимальные" представления, т.е. те, которые играют роль тождественных для $\mathfrak{g l}(V)$, осо- 
бенно в “классических" случаях $\mathfrak{p o}$ и $\mathfrak{b}$.

Теперь мы хотим обратить внимание читателя на следуюшее явление. Даже для “традищионной” супералгебры Пуассона $\mathfrak{p o}(2 n \mid m)$ сушествует несколько аналогов представления в пространстве Фока, отвечаюших различным нестандартным реализаииям $\mathfrak{p o}(2 n \mid m ; r)$ супералгебры $\mathfrak{p o}(2 n \mid m)$. Например, для $m=2 k$ эти реализации в терминах производящих функций от переменных $p_{i}, q_{i}(i=1, \ldots, n), \xi_{j}, \eta_{j}$ $(j=1, \ldots, k)$ задаются с помощью градуировки

$$
\begin{gathered}
\operatorname{deg} p_{i}=\operatorname{deg} q_{i}=\operatorname{deg} \xi_{j}=\operatorname{deg} \eta_{j}=1 \quad \forall i=1, \ldots, n \quad \text { и } j>r, \\
\operatorname{deg} \xi_{j}=0, \quad \operatorname{deg} \eta_{j}=2 \quad \text { для } 1 \leqslant j \leqslant r .
\end{gathered}
$$

Полное описание нестандартных реализаций - один из основных результатов классификации простых супералгебр Ли векторных полей (см. [38], [39]). Нам потребуются лишь некоторые из них (см. п. П.7). В настояшей статье мы подробно разбираем лишь стандартную реализацию (см. п. 4.2 , теорема 5 ).

Заметим, что хотя для нестандартных реализаций аналоги супералгебр Ли $\mathfrak{h} \mathfrak{e} \mathfrak{i}$ и $\mathfrak{a b}$ имеют разные размерности, присоединенные представления супералгебр Ли ро и $\mathfrak{b}$ являются "наименьшими” и единственными, в отличие от представлений соотношений (анти)коммутации.

1.6. Основные результаты. 1. Мы отмечаем, что супералгебра Ли $\mathfrak{h}(2 \mid 2)$ гамильтоновых векторных полей включается в однопараметрическое семейство $\mathfrak{h}_{\lambda}(2 \mid 2)$. Теорема 2 (см. ниже) показывает, как соотносятся деформации супералгебр Ли, задаваемых скобкой Пуассона и антискобкой. В частности, мы показываем, что алгера $\mathfrak{h}_{\lambda}(2 \mid 2)$ изоморфна переградуировке $\mathfrak{b}_{\lambda}(2 ; 2)$ супералгебры Ли $\mathfrak{b}_{\lambda}(2)$. Мы выводим отсюда новые исключительные квантования супералгебры Ли $\mathfrak{h}(2 \mid 2)$ гамильтоновых векторных полей и супералгебры Бюттен $\mathfrak{b}(2)$.

2. В качестве следствия мы получаем, что супералгебра Ли гамильтоновых векторных полей имеет больше квантований, чем супералгебра Пуассона. Казалось естественным ожидать, что супералгебра Ли $\mathfrak{h}(2 n \mid m)$ гамильтоновых векторных полей - фактор супералгебры $\mathfrak{p}(2 n \mid m)$ по центру - имеет в точности одно квантование, как и $\mathfrak{p o}(2 n \mid m)$. Эти надежды оправдались почти полностью, за исключением случая $(2 n, m)=(2,2)$, в котором параметры деформации принадлежат сингулярному супермногообразию, почти полностью описанному Кочетковым [1], [34]-[37]. Случай $(2 n, m)=(2,2)$ был пропушен им, несмотря на то что он должен был быть очевидным ввиду предшествовавшего результата [40]. В данной статье мы восполняем пробел в описании деформаций $\mathfrak{h}(2 n \mid m)$ и соотносим их с полным описанием квантований (деформаций) антискобки и ее фактора по центру $\mathfrak{l}(n)$.

3. В отличие от квантованной алгебры Пуассона $\mathfrak{p o}(2 n \mid 0)$, обладающей лишь одной реализацией посредством операторов рождения и уничтожения в фоковском пространстве, для квантованной супералгебры Бюттен $\mathfrak{b}(n)$ сушествуют $n+1$ различных фиоковских пространств, одно из которых конечномерно. Новым моментом в данном случае является необходимость нечетных параметров представлений. 
4. Приведенный выше результат тесно связан с описанием представлений аналогов соотношений коммутации для антискобки. Тогда как все нетривиальные неприводимые представления $\mathfrak{h} \mathfrak{e}(2 n \mid m)$ по существу эквивалентны и отличаются только значением центрального заряда, супералгебра Ли $\mathfrak{a} \mathfrak{b}(n)$, “нечетный” аналог $\mathfrak{h} \mathfrak{e}(2 n \mid m)$, имеет $n+1$ неприводимых представлений различной функциональной размерности. Ни одно из этих представлений не существует в наивном смысле: все они требуют нечетных параметров.

ЗАмЕчАния. А. Кочетков [1] доказал, что подалгебра $\mathfrak{s b}(n)$ бездивергентных ${ }^{1)}$ поливекторных полей (суперполей, гармонических относительно нечетного лапласиана) деформируема, и описал соответствующие коциклы (см. также [4]). Оказывается, что главная деформация супералгебры Ли $\mathfrak{b}(n)$, которую мы описьваем ниже, сохраняет $\mathfrak{s b}(n)$; это означает, что деформации $\mathfrak{s} \mathfrak{b}(n)$ имеют иную природу. Тем не менее ограничение квантования с $\mathfrak{b}(n)$ на $\mathfrak{s} \mathfrak{b}(n)$ нетривиально, Кочетков показал, что сушествуют и другие деформации $\mathfrak{s} \mathfrak{b}(n)$.

Б. Наш второй основной результат показывает, что только в одном случае супералгебра Ли гамильтоновых векторных полей может иметь больше квантований, чем соответствующая ей пуассонова супералгебра. Напротив, супералгебра Ли $\mathfrak{l}(n)$, нечетный аналог $\mathfrak{h}$, является более жесткой, чем $\mathfrak{b}(n):$ квантование порождает лиш деформацию $\mathfrak{l} \mathfrak{e}(n)$, других деформаций у $\mathfrak{k}(n)$ нет.

В. Чтобы упростить изложение и исследование, мы всюду ниже рассматриваем комплексные супералгебры Ли. Уместно заметить, что стандартная процедура перехода к вешественным формам всегда возможна, однако ее следует осушествлять чрезвычайно аккуратно (ср. теорему 9 из работы [41] с точным результатом Паркер и Сергановой [42] и с работой [39]).

Отметим, что использование теорем из [18] позволяет существенным образом сократить довольно объемные вычисления в контактных случаях [1], [43] (п. 3.3). Упрощения применимы к векторным полям с полиномиальными коэффициентами. Случай векторных полей, коэффициентами которых являются полиномы Лорана, и особенно их центральных расширений, сушественно отличается в техническом плане (по крайней мере, нам так кажется в настоящий момент); частичные результаты приведены в работе [36].

1.7. О двух недоразумениях. 1. Тенденция смешивать элементы супералгебры Ли $\mathfrak{p o}(2 n \mid m)$, индексированные функциями, с самими функциями (которые порождают ассоциативную и суперкоммутативную супералгебру относительно поточечного произведения) вносит путаницу и затрудняет исследование квантования в нашем смысле, т.е. деформации $\mathfrak{p o}(2 n \mid m)$ как супералгебры Ли. Чтобы избежать этого недоразумения, мы обозначаем ассоциативные (супер)алгебры латинскими буквами (например, $A$ ); то же пространство, рассматриваемое как некоторая (супер)алгебра Ли с (супер)скобкой $[x, y]$ вместо поточечного произведения $x y$ обозначается соответствующими готическими буквами $(\mathfrak{a})$ или индексом $L\left(A_{L}\right)$.

2. Общепринятое утверждение, что существует ассоциативная супералгебра $A$ такал, что $A_{L}=\mathfrak{p o}(2 n \mid m)$, неверно. Мы покажем это и дадим правильную формули-

\footnotetext{
1) В учебниках по физике, и особенно в трехмерии, такие поля называются соленоидальными.
} 
ровку в конце настоящего пункта. Однако для начала рассмотрим пример, иллюстрирующий суть проблемы.

В учебниках и статьях (см., например, [44]) можно встретить следующее описание алгебры Пуассона-Ли $\mathfrak{p o}(2 n)$ :

алгебра $\mathfrak{p o}(2 n)$ порождена (видимо, имеется в виду ассоциативная структура, хотя $\mathfrak{p o}(2 n)$ является алгеброй Ли) элементами $p_{i}$ и $q_{i}(i=1, \ldots, n)$, удовлетворяющими соотношениям

$$
\left\{p_{i}, q_{j}\right\}=i \hbar \delta_{i j}
$$

причем скобка должна удовлетворять правилу Лейбница

$$
\{f, g h\}=\{f, g\} h+g\{f, h\} .
$$

Очевидно, что правило (1.2) является не частью определения алгебры $\mathfrak{p o}(2 n)$, а одним из ее свойств - частным случаем производной Ли вдоль векторного поля, порожденного $f[26]$ (правило (1.2) является, однако, частью определения обобщенной пуассоновой структуры, определяемой некоторой вырожденной 2-формой, как, например, в [11]).

Уравнения (1.1) определяют гейзенбергову алгебру Ли hei $(2 n)$, пространством которой является $(2 n+1)$-мерное пространство $W \oplus \mathbb{C} z$, где $W$ есть $\operatorname{Span}(p, q)$ с невырожденной антисимметричной формой $B$ и $z$ принадлежит центру. Скобка задается соотношением (1.1) с правой частью, умноженной на $z$.

Наш нигилизм в отношении ассоциативных алгебр оправдан (как нам кажется) нашими результатами и некоторым прояснением общей картины. Но при решении других задач бывает необходимо рассматривать одновременно две структуры. Так Дринфельд при изучении интегрируемых систем даже ввел новое понятие алгебры Пуассона-Ли (с ассоциативным и лиевским умножениями, связанными друг с другом правилом Лейбница). Мы не рассматриваем это понятие в данной работе.

Используя $\mathfrak{h e i}(2 n)$, мы строим алгебру $\mathfrak{p o}(2 n)$ в два шага.

Шаг 1. Сначала мы рассматриваем ассоциативнуюалгебру $W e y l(2 n)=U(\mathfrak{h e i}(2 n))$. Поскольку нас интересуют неприводимые представления $\mathfrak{h} \mathfrak{e}(2 n)$, мы вспоминаем лемму Шура и фиксируем центральный заряд (вместо того чтобы рассматривать его как параметр), идентифицируя $z$ и $i \hbar$. Ассоциативная алгебра $\operatorname{diff}(n)$ дифференциальных операторов с полиномиальными коэффициентами на $n$-мерном пространстве может трактоваться как $U(\mathfrak{h e i}(2 n)) /(z-\hbar)$. Обе алгебры: и $W e y l(2 n)$, и ее фактор $\operatorname{diff}(n)$ - часто называют алгеброй Вейля. Обьчно из контекста можно догадаться, какая из двух имеется в виду.

Шаг 2. Пуассонова алгебра не изоморфна $\mathfrak{d i f f}(2 n)=\operatorname{diff}(2 n)_{L}$, но получается из $\mathfrak{d i f f}(2 n) e$ сжатием, т.е. переходом к пределу $\hbar \rightarrow 0$ после того, как мы положим, что $p=i \hbar \partial / \partial q$ в (1.1).

По-другому можно определить алгебру Пуассона как алгебру, изоморфную алгебpe $\operatorname{gr}(\mathfrak{d i f f}(2 n))$, т.е. градуированной алгебре Ли, ассоциированной с фильтрацией в $\mathfrak{d i f f}(2 n)$, возникающей из естественной фильтрации универсальной обертывающей алгебры $U(\mathfrak{h e i}(2 n))$. 
1.8. Связанные задачи. 1. Установив единственность квантования, желательно найти для него регулярную процедуру. На плоском пространстве существует несколько способов перейти от функции (т.е. символа, порождающего элемент алгебры Пуассона) к соответствующему оператору. Этими способами являются вейлевское, виковское, $p q$-квантование и т.д. Теорема единственности утверждает, что все они по сушеству эквивалентны. Описание квантований на пространствах, которые локально-эквивалентны (в смысле $G$-структур) классическим областям, было недавно предпринято в работах [45], [46].

2. Задача описания образующих и определяюших соотношений po $(2 n)$ как алгебры Ли (обсуждавшаяся в п. 1.7) решена в [47] (по поводу суперизации и нерешенных задач см. $[48])$.

3. Задача $q$-квантования конечномерных пуассоновых супералгебр исследовалась в работах [49], [50].

\section{2. ДЕФОРМАЦИИ СУПЕРАЛГЕБРЫ БЮТТЕН И ЕЕ ПОДАЛГЕБР}

Все необходимые определения приведены в приложении.

2.1. Главная деформация [40], [51]. Из определения скобки Бюттен (см. ниже (П.10)) следует, что сушествует переградуировка (а именно, $\mathfrak{b}(n ; n)$, определяемая условиями $\operatorname{deg} \xi_{i}=0, \operatorname{deg} q_{i}=1$ для всех $\left.i\right)$, при которой супералгебра $\mathfrak{b}(n)$, изначально имеюшая глубину 2 , принимает вид

$$
\mathfrak{g}=\bigoplus_{i \geqslant-1} \mathfrak{g}_{i}
$$

где $\mathfrak{g}_{0}=\mathfrak{v e c t}(0 \mid n)$ и $\mathfrak{g}_{-1} \cong \Pi(\mathbb{C}[\xi])$. Заменим $\mathfrak{v e c t}(0 \mid n)$-модуль $\mathfrak{g}_{-1}$ функций (с обрашенной четностью) модулем $\lambda$-плотностей, т.е. положим $\mathfrak{g}_{-1} \cong \Pi\left(\operatorname{Vol}(0 \mid n)^{\lambda}\right)$ (см. п. П.6), где действие алгебры Ли $\mathfrak{g}_{0}=\mathfrak{v e c t}(0 \mid n)$ определяется для любых $D \in \mathfrak{g}_{0}$ и $f \in \mathbb{C}[\xi]$ формулами

$$
L_{D}\left(f \operatorname{vol}_{\xi}^{\lambda}\right)=\left(D(f)+(-1)^{p(D) p(f)} \lambda f \operatorname{div} D\right) \cdot \operatorname{vol}_{\xi}^{\lambda}, \quad p\left(\operatorname{vol}_{\xi}^{\lambda}\right)=\overline{1} .
$$

Определим $\mathfrak{b}_{\lambda}(n ; n)$, деформацию $\mathfrak{b}(n ; n)$, как картановское продолжение пары:

$$
\mathfrak{b}_{\lambda}(n ; n):=\left(\mathfrak{g}_{-1}, \mathfrak{g}_{0}\right)_{*}=\left(\Pi\left(\operatorname{Vol}(0 \mid n)^{\lambda}\right), \mathfrak{v e c t}(0 \mid n)\right)_{*} .
$$

Эти алгебры $\mathfrak{b}_{\lambda}(n ; n)$ при всевозможных $\lambda$ образуют главную деформацию (хотя эта деформация и называется главной, она не является квантованием скобки Бюттен; о квантовании см. п. 2.2).

Деформация $\mathfrak{b}_{\lambda}(n)$ супералгебры $\mathfrak{b}(n)$ является переградуировкой алгебры $\mathfrak{b}_{\lambda}(n ; n)$, которую можно описать следующим образом. Положим

$$
\mathfrak{b}_{a, b}(n)=\left\{M_{f} \in \mathfrak{m}(n) \mid a \operatorname{div} M_{f}=(-1)^{p(f)} 2(a-b n) \frac{\partial f}{\partial \tau}\right\} .
$$


Принимая во внимание явный вид (см. ниже (П.13)) дивергенции поля $M_{f}$, мы видим, что

$$
\begin{aligned}
\mathfrak{b}_{a, b}(n) & =\left\{M_{f} \in \mathfrak{m}(n) \mid(b n-a E) \frac{\partial f}{\partial \tau}=a \Delta f\right\}= \\
& =\left\{D \in \operatorname{vect}(n \mid n+1) \mid L_{D}\left(\operatorname{vol}_{q, \xi, \tau}^{a} \alpha_{0}^{a-b n}\right)=0\right\} .
\end{aligned}
$$

Непосредственно проверяется, что $\mathfrak{b}_{a, b}(n)$ есть просто другое обозначение для $\mathfrak{b}_{\lambda}(n)$ при $\lambda=2 a / n(a-b)$. Это показывает, что в действительности $\lambda$ пробегает проективную прямую $\mathbb{C} P^{1}$, а не $\mathbb{C}$.

Имеют место следующие изоморфизмы: $\mathfrak{b}_{n b, b}(n) \cong \mathfrak{s m}(n), \mathfrak{b}_{a,-a}(2 ; 2) \cong \mathfrak{b}_{1 / 2}(2 \mid 2) \cong$ $\mathfrak{h}(2 \mid 2)$ и $\mathfrak{b}_{-a,-b}(n) \cong \mathfrak{b}_{a, b}(n)$. Кроме того, $\mathfrak{b}_{\lambda}(2) \simeq \mathfrak{h}_{\lambda}(2 \mid 2)$, где $\mathfrak{h}_{\lambda}(2 \mid 2)-$ деформация $\mathfrak{h}(2 \mid 2)$, описанная в разделе 3 .

Супералгебры Ли $\mathfrak{b}_{\lambda}(n)$ просты при $n>1$ и $\lambda \neq 0,1, \infty$. Понятно, что супералгебры $\mathfrak{b}_{\lambda}(n)$ с различными $\lambda$ не изоморфны.

У супералгебры Ли $\mathfrak{b}(n)=\mathfrak{b}_{0}(n)$ есть $\varepsilon$-мерный, т.е. $(0 \mid 1)$-мерный, центр, фактор по которому - простая алгебра. При $\lambda=1, \infty$ супералгебры Ли $\mathfrak{b}_{\lambda}(n)$ также не просты: они содержат идеалы, которые мы обозначаем $\mathfrak{b}_{1}^{\circ}(n)$ и $\mathfrak{b}_{\infty}^{\circ}(n)$, коразмерностей $\varepsilon^{n}$ и $\varepsilon^{n+1}$, соответственно (см. [39]). Соответствуюшие точные последовательности имеют вид

$$
\begin{aligned}
& 0 \rightarrow \mathbb{C} \cdot M_{1} \rightarrow \mathfrak{b}(n) \rightarrow \mathfrak{l e}(n) \rightarrow 0 \\
& 0 \rightarrow \mathfrak{b}_{1}^{\circ}(n) \rightarrow \mathfrak{b}_{1}(n) \rightarrow \mathbb{C} \cdot M_{\xi_{1} \ldots \xi_{n}} \rightarrow 0, \\
& 0 \rightarrow \mathfrak{b}_{\infty}^{\circ}(n) \rightarrow \mathfrak{b}_{\infty}(n) \rightarrow \mathbb{C} \cdot M_{\tau \xi_{1} \ldots \xi_{n}} \rightarrow 0 .
\end{aligned}
$$

Ясно, что для исключительных значений $\lambda=0,1, \infty$ деформации $\mathfrak{b}_{\lambda}(n)$ следует изучать наиболее тщательно. Усилия будут вознаграждены: в каждой исключительной точке есть дополнительные деформации. Наличие исключительной деформации при $\lambda=-1$ остается необъясненным.

ЗАмечания. 1. Скобка Схоутена была первоначально определена на суперпространстве поливекторных полей на супермногообразии, т.е. на суперпространстве сечений внешней алгебры (над алгеброй $\mathcal{F}$ функций) касательного расслоения $\Gamma\left(\Lambda^{\bullet}(T(M))\right) \cong \Lambda_{\mathcal{F}}^{\bullet}(\operatorname{Vect}(M))$. Точная формула для скобки Схоутена имеет вид

$$
\begin{aligned}
{\left[X_{1} \wedge \cdots \wedge X_{k}, Y_{1} \wedge \cdots \wedge Y_{l}\right]=} & \sum_{i, j}(-1)^{i+j}\left[X_{i}, Y_{j}\right] \wedge X_{1} \wedge \cdots \wedge \widehat{X}_{i} \wedge \cdots \wedge X_{k} \wedge \\
& \wedge Y_{1} \wedge \cdots \wedge \widehat{Y}_{j} \wedge \cdots \wedge Y_{l}
\end{aligned}
$$

где, как обычно, крышка над переменной означает ее пропуск. С помошью правила знаков мы легко обобщаем формулу (2.6) на случай, когда $M$ заменяется на супермногообразие $\mathcal{M}$. Связь суперверсии фомулы (2.6) с формулой (П.10) следующая. Пусть $x$ и $\xi$ суть четные и нечетные координаты на $\mathcal{M}$. Полагая $\theta_{i}=\Pi\left(\partial / \partial x_{i}\right)=\check{x}_{i}$, $q_{j}=\Pi\left(\partial / \partial \xi_{j}\right)=\check{\xi}_{j}$, мы получим отождествление скобки Схоутена поливекторных полей на $\mathcal{M}$ со скобкой Бюттен функций на супермногообразии $\check{\mathcal{M}}$, координатами которых являются $x, \xi$ и $\breve{x}, \check{\xi}$, причем любые преобразования координат $x, \xi$ порождают соответствуюшие преобразования координат $\check{x}, \check{\xi}$. 
2. Грозман [51] классифицировал все инвариантные дифференциальные операторы, действующие на пространствах сечений тензорных полей на любом многообразии. Он также ввел однопараметрическое семейство деформаций скобки Схоутена, связанное с тем, что мы называем “главной деформацией”, а именно он ввел оператор

$$
\begin{aligned}
X \operatorname{vol}^{\mu}, Y \operatorname{vol}^{\nu} \mapsto & \left((\nu-1)(\mu+\nu-1) \operatorname{div} X \cdot Y+(-1)^{p(X)}(\mu-1)(\mu+\nu-1) X \operatorname{div} Y-\right. \\
& -(\mu-1)(\nu-1) \operatorname{div}(X Y)) \operatorname{vol}^{\mu+\nu},
\end{aligned}
$$

где дивергенцию поливекторного поля лучше всего описать с помощью координат $(x, \check{x})$ на супермногообразии $\check{M}$, ассоциированном с супермногообразием $M$ (см. ниже формулу (П.15)).

3. Введенная Грозманом супералгебра Ли на скрученных поливекторных полях на $M$, задаваемая формулой (2.7), может быть реализована как подалгебра в супералгебре Ли $\mathfrak{s} \mathfrak{b}(n+1)$ бездивергентных поливекторных полей на $M \times \mathbb{R}_{+}$или как супералгебра Ли функций (относительно скобки Бюттен) на ассоциированном супермногообразии с координатами $\check{x}, \check{\xi}$. Точная формула имеет вид

$$
X \operatorname{vol}^{\lambda} \mapsto t^{-\lambda} X+\frac{1}{\lambda-1} t^{-\lambda+1} \frac{\partial}{\partial t} \wedge \operatorname{div}(X) .
$$

В терминах п. П.4 правая часть формулы (2.8) есть

$$
t^{-\lambda} f(x, \xi)+\frac{1}{\lambda-1} t^{-\lambda+1} \check{t} \Delta(f) .
$$

2.2. Краткое изложение работ [34], [1], [4]: квантования. Деформацию $\mathfrak{b}_{\lambda}(n)$ супералгебры $\mathfrak{b}(n)$, которая связывает $\mathfrak{b}(n)$ с $\mathfrak{s m}(n)$, мы назвали главной. Другие деформации, называемые сингулярными, не менее интересны. Особый интерес представляют те из них, которые получаются при $\lambda=0$ и (для $n=2)$ при $\lambda=1 / 2$ : они являются квантованиями.

Положим $H=H^{2}(\mathfrak{g} ; \mathfrak{g})$ при $\mathfrak{g}=\mathfrak{b}_{\lambda}(n)$. Напомним [18], что пространство $H$ обычно отождествляют с касательным пространством к сингулярному супермногообразию параметров деформаций алгебры $\mathfrak{g}$ в точке, отвечающей самой алгебре $\mathfrak{g}$. Каждой глобальной деформации супералгебры Ли $\mathfrak{g}$ соответствует некоторый коцикл - элемент пространства $H$. Обратное, вообше говоря, неверно. Коцикл определяет лишь локальную деформацию, которая может и не продолжаться до глобальной.

В следующей теореме мы используем реализацию элементов супералгебры Ли $\mathfrak{b}_{\lambda}(n)$ производящими функциями $f \in \mathbb{C}\left[q_{1}, \ldots, q_{n}, \xi_{1}, \ldots, \xi_{n}, \tau\right]$ (см. п. П.3). Под $\operatorname{deg}_{\overline{1}} f$ мы понимаем степень полинома $f$ относительно только нечетных аргументов. Мы используем также стандартные мультииндексные обозначения $q^{k}=q_{1}^{k_{1}} \ldots q_{n}^{k_{n}}$ и $|k|=\sum k_{i}$ для мультииндекса $k=\left(k_{1}, \ldots, k_{n}\right)$.

Tеорема 1. 1. Размерность пространства $H$ равна $\operatorname{dim} H=(1 \mid 0)$ для $\mathfrak{g}=$ $\mathfrak{b}_{\lambda}(n)$ при $n>2$, за исключением случаев $\lambda=0,-1,1, \infty$. Eсли же $n=2$, то $\operatorname{dim} H \neq(1 \mid 0)$ еще и при $\lambda=1 / 2$.

2. При исключительных значениях $\lambda$, указанньх в п. 1, имеем: 
$\operatorname{dim} H=(2 \mid 0)$ при $\lambda= \pm 1$ и нечетном $n$, или $\lambda=\infty$ и четном $n$, или $\lambda=1 / 2$ и $n=2$;

$\operatorname{dim} H=(1 \mid 1)$ при $\lambda=0$ и любом $n$, или $\lambda=\infty$ и нечетном $n$, или $\lambda= \pm 1 u$ четном $n$.

Соответствующие кочикль $C$ определяются ненулевыми значениями на производящих функциях $f$ и $g$, приведенными в табл. 1.

Таблица 1

\begin{tabular}{|c|c|c|}
\hline $\mathfrak{b}_{\lambda}(n)$ & $p(C)$ & $C(f, g)$ \\
\hline $\mathfrak{b}_{0}(n)$ & $\overline{1}$ & $(-1)^{p(f)}\left(d_{\overline{1}}(f)-1\right)\left(d_{\overline{1}}(g)-1\right) f g$ \\
\hline $\mathfrak{b}_{-1}(n)$ & $n+1(\bmod 2)$ & $\begin{array}{c}f=q^{k}, \quad g=q^{l} \\
C=(4-|k|-|l|) q^{k+l} \xi_{1} \ldots \xi_{n}+\tau \Delta\left(q^{k+l} \xi_{1} \ldots \xi_{n}\right)\end{array}$ \\
\hline $\mathfrak{b}_{1}(n)$ & $n+1(\bmod 2)$ & $C=\left\{\begin{array}{cc}f=\xi_{1} \ldots \xi_{n}, \quad g \text { любое } \\
\left(d_{\overline{1}}(g)-1\right) g \quad \text { nри } g \neq a f, \quad a \in \mathbb{C}, \\
2(n-1) f \quad \text { nри } g=f \text { и четном } n\end{array}\right.$ \\
\hline $\mathfrak{b}_{\infty}(n)$ & $n(\bmod 2)$ & $C= \begin{cases}f=\tau \xi_{1} & \ldots \xi_{n}, \quad g \text { любое } \\
\left(d_{\overline{1}}(g)-1\right) g & \text { nри } g \neq \text { af, } \quad \text { a } \in \mathbb{C}, \\
2 f & \text { nри } g=f \text { и нечетном } n\end{cases}$ \\
\hline
\end{tabular}

Кочикл на $\mathfrak{b}_{1 / 2}(2) \simeq \mathfrak{h}(2 \mid 2 ; 1)$ (последняя алгебра есть переградуировка $\mathfrak{h}(2 \mid 2)$, см. n. П.7) индуцирован на $\mathfrak{h}(2 \mid 2)=\mathfrak{p o}(2 \mid 2) / \mathbb{C}$ объчным квантованием po $(2 \mid 2)$ : сначала мы квантуем ро, а затем факторизуем по иентру (порожсенному константами).

3. Пространство Н диагонализуемо относительно подалгебры Картана в $\mathfrak{d e r} \mathfrak{g}$; кочикл $M$, соответствующий главной деформации, является одним из собственных векторов. В исключительных случаях, описанных в $n .2, к о г д а ~ \operatorname{dim} H>1$, второй собственный вектор - коцикл $C$ - соответствует сингулярной деформации. Для всех остальных коииклов, т.е. для коциклов вида $k M+l C$ c $k l \neq 0$, локальные деформачии, определяемые этими кочиклами, не продолжсаются до глобальных.

Все исключительные деформации скобки $\{\cdot, \cdot\}_{\text {old }}$ в $\mathfrak{b}_{\lambda}(n)$ (кроме случая $\lambda=1 / 2$ u $n=2$ ) имеют очень простой вид даже при четном параметре $\hbar:$

$$
\{f, g\}_{\hbar}^{\text {sing }}=\{f, g\}_{\text {old }}+\hbar \cdot C(f, g) \quad \text { npu } \bumpeq ю б b l x \quad f, g \in \mathfrak{b}_{\lambda}(n)
$$


ЗАМЕчАниЕ. К. Рожэ заметил, что сингулярная деформация (квантование) $\mathfrak{b}_{0}(n)=$ $\mathfrak{b}(n)$ совпадает с точностью до знака с внешним произведением двух 1-коциклов - дифференцирований $f \mapsto\left(d_{\overline{1}}(f)-1\right) f$. Он также посоветовал нам напомнить читателям, что коцикл на $\mathfrak{b}_{1 / 2}(2) \simeq \mathfrak{h}(2 \mid 2)$, индуцированный квантованием $\mathfrak{p o}(2 \mid 2)$, является прямой суперизацией хорошо известного коцикла Вея [52].

Поскольку элементы $\mathfrak{b}_{\lambda}(n)$ реализованы полиномами от $\tau, q$ и $\xi$, удовлетворяюшими одному соотношению с нечетной левой частью, содержашему $\tau$, то естественно предположить, что скобка может быть, по крайней мере при обших значениях $\lambda$, выражена только через $q$ и $\xi$. Действительно, имеется явная формула (в которой $\{f, g\}_{B . b .}-$ это обычная антискобка):

$$
\left\{f_{1}, f_{2}\right\}_{\lambda}^{\text {main }}=\left\{f_{1}, f_{2}\right\}_{B . b .}+\lambda\left(c_{\lambda}\left(f_{1}, f_{2}\right) f_{1} \Delta f_{2}+(-1)^{p\left(f_{1}\right)} c_{\lambda}\left(f_{2}, f_{1}\right)\left(\Delta f_{1}\right) f_{2}\right),
$$

где

$$
c_{\lambda}\left(f_{1}, f_{2}\right)=\frac{\operatorname{deg} f_{1}-2}{2+\lambda\left(\operatorname{deg} f_{2}-n\right)}
$$

и $\operatorname{deg}$ вычисляется относительно стандартной градуировки $\operatorname{deg} q_{i}=\operatorname{deg} \xi_{i}=1$.

\section{3. ГЛАВНЫЕ ДЕФОРМАЦИИ $\mathfrak{h}(2 \mid 2)$}

Формула (2.2) интерпретирует $\mathfrak{b}_{\lambda}(n)$ как супералгебру Ли, сохраняюшую сложный тензор $\operatorname{vol}_{q, \xi, \tau}^{a} \alpha_{0}^{a-b n}$. При $n=2$, следуя Шмелеву (см. [40]), мы определяем деформацию $\mathfrak{h}_{\lambda}(2 \mid 2)$ супералгебры Ли $\mathfrak{h}(2 \mid 2)$ гамильтоновых векторных полей, сохраняюшую либо псевдодифференциальную форму

$$
d \eta^{\frac{2 \lambda-1}{1-\lambda}}(\lambda d q d p+(1-\lambda) d \xi d \eta)
$$

либо, что эквивалентно, псевдодифференциальную форму

$$
d \eta^{\frac{1}{\lambda}-2}(d q d p+d \xi d \eta)
$$

Сравнение неположительных членов $\mathbb{Z}$-градуировок показывает, что $\mathfrak{h}_{\lambda}(2 \mid 2) \cong$ $\mathfrak{b}_{\lambda}(2 ; 2)$.

Приведем явную реализацию элементов $D \in \mathfrak{b}_{\lambda}(2 ; 2) \subset \mathfrak{v e c t}(2 \mid 2)$ в терминах элементов из $\mathfrak{h}(2 \mid 2)$ с добавкой, зависяшей от $\lambda$. Мы предполагаем, что гамильтонианы, производящие гамильтоновы векторные поля из $\mathfrak{h}(2 \mid 2)$, принадлежат $\mathbb{C}[p, q, \xi, \eta]$. 


\section{1. Явное описание главной деформации.}

Teорема 2. Пусть $\hbar(\lambda)=(2 \lambda-1) / \lambda$. Элемент $D \in \mathfrak{v e c t}(2 \mid 2)$ принадлежит $\mathfrak{b}_{\lambda}(2 ; 2)$ тогда и только тогда, когда $D$ имеет вид

$$
D=D_{f}=H_{f}+\hbar(\lambda) W_{f}
$$

¿əe

$$
W_{f}=\left(\int_{0}^{p} \frac{\partial}{\partial \eta} \frac{\partial f}{\partial \xi} d p\right) \partial_{p}+(-1)^{p(f)} \frac{\partial f}{\partial \xi} \partial_{\eta}
$$

для некоторого $f \in \mathbb{C}[p, q, \xi, \eta]$. Кроме того, для $f, g \in \mathbb{C}[p, q, \xi, \eta]$ имеем

$$
\left[D_{f}, D_{g}\right]=D_{\{f, g\}}+\hbar(\lambda) D_{c(f, g)},
$$

ฉде

$$
\begin{aligned}
c(f, g)=- & \frac{\partial f}{\partial p} \int_{0}^{p} \frac{\partial}{\partial \eta} \frac{\partial g}{\partial \xi} d p+\frac{\partial g}{\partial p} \int_{0}^{p} \frac{\partial}{\partial \eta} \frac{\partial f}{\partial \xi} d p+ \\
& +\frac{\partial}{\partial \eta}\left(\int_{(0, q)}^{(p, q)}\left((-1)^{p(f)} \frac{\partial f}{\partial p} \frac{\partial g}{\partial \xi}-\frac{\partial f}{\partial \xi} \frac{\partial g}{\partial p}\right) d p+\right. \\
& \left.+\left.\int_{(0,0)}^{(0, q)}\left((-1)^{p(f)} \frac{\partial f}{\partial q} \frac{\partial g}{\partial \xi}-\frac{\partial f}{\partial \xi} \frac{\partial g}{\partial q}\right)\right|_{p=0} d q\right)+ \\
& +\left.\xi \frac{\partial}{\partial \xi}\left((-1)^{p(f)} \frac{\partial f}{\partial \xi} \frac{\partial g}{\partial \eta}+\frac{\partial f}{\partial \eta} \frac{\partial g}{\partial \xi}\right)\right|_{p=0, q=0} .
\end{aligned}
$$

Заметим, что формула

$$
\left[H_{f}, H_{g}\right]_{\mathrm{new}}=H_{\{f, g\}_{P . b .}}+\hbar(\lambda) \cdot H_{c(f, g)}
$$

определяет некоторую деформацию $\mathfrak{h}(2 \mid 2)$ (являюшуюся главной деформацией $\left.\mathfrak{b}_{1 / 2}(2)\right)$, но (и это согласуется с результатами [14]) формула

$$
\{f, g\}_{\text {new }}=\{f, g\}+\hbar(\lambda) \cdot c(f, g)
$$

не определяет деформацию po $(2 \mid 2)$, поскольку новая скобка не удовлетворяет тождеству Якоби.

3.2. Деформации $\mathfrak{g}=\mathfrak{b}_{1 / 2}(n ; n)$. Понятно, что $\mathfrak{g}_{-1}$ изоморфна $\Pi(\sqrt{\mathrm{Vol}})$. Следовательно, имеют место вложения

$$
\mathfrak{b}_{1 / 2}(n ; n) \subset \begin{cases}\mathfrak{h}\left(2^{n-1} \mid 2^{n-1}\right) & \text { при четном } n \\ \mathfrak{l}\left(2^{n-1}\right) & \text { при нечетном } n .\end{cases}
$$

Тогда возникает желание определить те квантования $\mathfrak{g}$, которые не рассматривал Кочетков, как композиции вложений (3.2) и последуюших квантований. Так можно поступить при $n=2$, когда (3.2) есть не просто вложение, но и изоморфизм; мы получаем дополнительное квантование антискобки, описанное в теореме 1: сначала деформируем ее в точку $\lambda=1 / 2$ вдоль главной деформашии и затем квантуем ее как фактор супералгебры Пуассона. Однако эта процедура не дает новых алгебр при $n=2 k>2$. 
ТЕОрема 3. Если $n=2 k>2$, то образ $\mathfrak{b}_{1 / 2}(n ; n)$ при вложсениях (3.2) недеформируем при квантованиях обгемлющей алгебры.

Доказательство этой теоремы непосредственно, но вычисления громоздкие, поэтому оно будет помещено только в сети Интернет.

3.3. Общие алгебры недеформируемы. Недеформируемость контактных серий была установлена с помощью громоздких вычислений Кочетковым [1] для серии $\mathfrak{m}$ и Шмелевым [43] для серии k. Этот факт, однако, является примером общих утверждений о когомологиях коиндуцированных модулей [18] и непосредственно следует из более поздних вычислений когомологий $\mathfrak{g l}(m \mid n), \mathfrak{o s p}(m \mid 2 n)$ и $\mathfrak{p} \mathfrak{e}(n)$ (cм. [53], [18]) и того, что общие алгебры $\mathfrak{v e c t}, \mathfrak{k}$ и $\mathfrak{m}$, как модули над самими собой, имеют вид обобщенных тензорных полей (см. п. П.6):

$\mathfrak{v e c t}(m \mid n)=T\left(\operatorname{id}_{\mathfrak{g l}(m \mid n)}\right) ; \quad \mathfrak{k}(2 m+1 \mid n)=T\left(\operatorname{id}_{\mathfrak{c o s p}(n \mid 2 m)}\right) ; \quad \mathfrak{m}(n)=T\left(\operatorname{id}_{\mathfrak{c p e}(n)}\right)$.

Следовательно, присоединенные модули коиндуцированы, и справедлива следующая

ТЕОРЕма 4. При $\mathfrak{g}=\mathfrak{v e c t}(m \mid n), \mathfrak{k}(2 m+1 \mid n) u \mathfrak{m}(n)$ имеeт место

$$
H^{2}(\mathfrak{g} ; \mathfrak{g}) \simeq H^{2}\left(\mathfrak{g}_{0} ; \mathrm{id}_{\mathfrak{g}_{0}}\right)=0
$$

\section{4. ПРЕДСТАВЛЕНИЯ $\mathfrak{h} \mathfrak{e}(2 n \mid m), \mathfrak{a b}(n)$}

Заметим, что только для стандартной реализации (см. п. П.7) связь между соотношениями (анти)коммутации (представленными элементами отрицательной степени из супералгебр Пуассона или Бюттен) и самими супералгебрами Пуассона или Бюттен такая же, как для алгебры Ли ро $(2 n)$ (чтобы увидеть особенности нестандартной реализации наиболее отчетливо, рассмотрите конечномерный случай, скажем po $(0 \mid 2 n)$ с градуировкой $\operatorname{deg} \xi_{i}=0, \operatorname{deg} \eta_{i}=1$ при всех $\left.i\right)$.

\section{1. Язык $C$-точек.}

ЛЕмма [54]. 1. Пусть $V$ - некоторое векторное суперпространство, а $P_{V}(C)=(V \otimes C)_{\overline{0}}-$ множество его $C$-точек. Тогда $V \simeq W$ в том и только в том случае, когда $P_{V}(C) \simeq P_{W}(C)$ для всех суперкоммутативных супералгебр $C$.

2. Пусть $\mathfrak{g} u \mathfrak{h}$ - супералгебры Ли. Тогда $\mathfrak{g} \simeq \mathfrak{h}$ в том и только в том случае, когда для всех суперкоммутативных супералгебр $C \quad P_{\mathfrak{g}}(C) \simeq P_{\mathfrak{h}}(C)$, как алгебры $\pi u$.

3. Пусть $V$ и $W-д в а ~ м о д у л я ~ н а д ~ \mathfrak{~ . ~ О н и ~ и з о м о р ф н ь и ~ т о г д а ~ и ~ т о л ь к о ~ т о г д а , ~}$ когда для всех суперкоммутативных супералгебр $C P_{V}(C) \simeq P_{W}(C)$, как модули над $P_{\mathfrak{g}}(C)$.

4. Достаточно проверять вышеприведенные условия для $C=\Lambda(N)$ при достаточно больших $N$.

ЗАмечАниЕ. Заменять $N$ на $\infty$ в утверждении 4 леммы, как это предлагал Березин, не стоит: хотя вместо бесконечно большого набора условий нам придется проверить только одно, мы приобретем серьезные топологические сложности [55]. 
4.2. Неприводимые представления супералгебры $\mathfrak{h} \mathfrak{e}(2 n \mid m)$ и ее аналогов. Следуюшее утверждение при $M=0$ и его аналог - утверждение 1 из теоремы 6 являются частными случаями одного результата Сергеева [56], исправляюшего теорему 7 из работы [41].

ТЕОРема 5. Представим суперпространство $\mathfrak{h e i}(2 n \mid m)$ как $W \oplus \mathbb{C} z$, где $W$ снабжено формой $B$ (см. n. П.5.1 и П.5.2), и пусть $W=V \oplus V^{*}$, если $m=2 k$, или $W=V \oplus V^{*} \oplus U$, если $m=2 k+1$, дде $V u V^{*}$ изотропны относительно $B u$ имеют размерность $n \mid k$. Тогда любое неприводимое косоэрмитово представление $\mathfrak{h e i}(2 n \mid m)$ (над полем $\mathbb{C})$ әквивалентно представлению в следующих фоковских суперпространствах: $\mathcal{F}_{\hbar} \simeq \mathbb{C}[V]$, если $m=2 k$, и это представление $G$-типа, или $\mathcal{F}_{\hbar} \simeq \mathbb{C}[V \oplus U]$, если $m=2 k+1$, и әто представление $Q$-типа, т.е. $\mathfrak{h e i}(2 n \mid 2 k+1)$ вкладывается в $\mathfrak{q}\left(\mathcal{F}_{\hbar}\right)$. ЦЦентр z супералгебры $\mathfrak{h e i}(2 n \mid m)$ действует на $\mathcal{F}_{\hbar}$ как умножение на $і \hbar, \hbar \in \mathbb{R}$.

4.3. Неприводимые представления $\mathfrak{a} \mathfrak{b}(n)$. Напомним, что $\mathfrak{a} \mathfrak{b}(n)=W \oplus \mathbb{C} z($ см. п. П.5.2). Пусть $W$ является линейной оболочкой четных элементов $q_{1}, \ldots, q_{n}$ и нечетных элементов $\theta_{1}, \ldots, \theta_{n}$.

ТЕОРема 6. 1. Над любой коммутативной алгеброй с нулевой нечетной частью супералгебра $\mathfrak{a b}(n)$ имеет только два неприводимых представления: (1|0)мерный тривиальный модуль $\mathbf{1}$ и П(1).

2. Пусть $C$ - суперкоммутативная супералгебра с $C_{\overline{1}} \neq 0$ и пусть $\xi \in C_{\overline{1}}$. Существуют $n+1$ различных неприводимых $\mathfrak{a b}(n ; C)$-модулей $\mathcal{F}_{i}$, соответствующих нечетным параметрам, описывающим пространство, касательное к тривиальному представлению 1. Именно, для ненулевого вакуумного вектора $v$ положим $z v=\xi v u$

$$
q_{1} v=\cdots=q_{i} v=\theta_{i+1} v=\cdots=\theta_{n} v=0 \quad \text { npu } \quad i=0,1, \ldots, n
$$

и определим

$$
\mathcal{F}_{i}=\operatorname{ind}_{\operatorname{Span}\left(q_{1}, \ldots, q_{i}, \theta_{i+1}, \ldots, \theta_{n} ; z\right)}^{\mathfrak{a} \mathfrak{b}(n)}(C v) \simeq C\left[\lambda_{1}, \ldots, \lambda_{i} ; x_{i+1}, \ldots, x_{n}\right] .
$$

Явная реализачия операторов имеет вид

$$
\begin{array}{rlrl}
q_{1} & \mapsto \xi \frac{\partial}{\partial \lambda_{1}}, \ldots, q_{i} \mapsto \xi \frac{\partial}{\partial \lambda_{i}}, & q_{i+1} \mapsto x_{i+1}, \ldots, q_{n} \mapsto x_{n}, \\
\theta_{1} \mapsto \lambda_{1}, \ldots, \theta_{i} \mapsto \lambda_{i}, & \theta_{i+1} \mapsto-\xi \frac{\partial}{\partial x_{i+1}}, \ldots, \theta_{n} \mapsto-\xi \frac{\partial}{\partial x_{n}} .
\end{array}
$$

Итак, как суперпространства,

$$
\begin{gathered}
\mathfrak{p o}(2 n \mid 2 m) \simeq \mathbb{C}\left[q, \frac{\partial}{\partial q}, \xi, \frac{\partial}{\partial \xi}\right] \simeq U(\mathfrak{h e i}(2 n \mid 2 m)) /(z-\hbar), \\
\Pi(\mathfrak{b}(n) \otimes C[\xi]) \simeq(U(\mathfrak{a} \mathfrak{b}(n)) \otimes C[\xi]) /(z-\xi),
\end{gathered}
$$

где для антискобки мы должны ввести суперкоммутативную супералгебру $C$, чтобы учесть нечетные параметры. 
ПРИЛОЖКЕНИЕ

\section{П.1. Элементы линейной алгебры в суперпространствах}

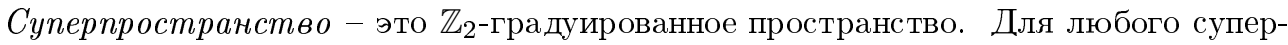
пространства $V=V_{\overline{0}} \oplus V_{\overline{1}}$ символом $\Pi(V)$ обозначим копию того же пространства со сдвинутой четностью, т.е. $(\Pi(V))_{\bar{i}}=V_{\bar{i}+\overline{1}}$. Суперразмерность суперпространства $V$ определяется равенством $\operatorname{dim} V=p+q \varepsilon$, где $\varepsilon^{2}=1$ и $p=\operatorname{dim} V_{\overline{0}}, q=\operatorname{dim} V_{\overline{1}}$ (обычно $\operatorname{dim} V$ указывается парой $p \mid q$, это обозначение делает полезную формулу $\operatorname{dim} V \otimes$ $W=\operatorname{dim} V \operatorname{dim} W$ загадочной, но если использовать $\varepsilon$, то она очевидна).

Структура суперпространства на $V$ индуцирует структуру суперпространства на $\operatorname{End}(V)$. Базис суперпространства всегда состоит из однородных векторов. Если $p_{i}$ обозначает четность $i$-го базисного вектора, то матричная единица $E_{i j}$ имеет четность $p_{i}+p_{j}$, и скобка суперматриц определяется с помощью правила знаков:

если әлемент четности р перемещается мимо әлемента четности q, то появляется множитель $(-1)^{p q}$; формуль, определенные на однородных әлементах, расииряются на произвольные әлементы по линейности.

Приведем два примера применения правила знаков. Полагая $[X, Y]=X Y-$ $(-1)^{p(X) p(Y)} Y X$, мы получаем определение суперкоммутатора, на основе которого вводятся понятия суперкоммутативной супералгебры и супералгебры Ли, в которой наряду с суперантисимметричностью выполняется супертождество Якоби, т.е. тождество Якоби, подправленное по правилу знаков. Супердифференцирование супералгебры $A-$ это линейное отображение $D: A \rightarrow A$, удовлетворяющее правилу Лейбница (и правилу знаков)

$$
D(a b)=D(a) b+(-1)^{p(D) p(a)} a D(b) .
$$

В частности, пусть $A=\mathbb{C}[x]$ - это свободная суперкоммутативная супералгебра полиномов от $x=\left(x_{1}, \ldots, x_{n}\right)$, в которой суперструктура определена четностями аргументов: $p_{i}=p\left(x_{i}\right)$. Частные производные определяются с помощью суперправила Лейбница формулами $\partial x_{i} / \partial x_{j}=\delta_{i j}$. Понятно, что множество $\mathfrak{d e r} A$ всех супердифференцирований $A$ - это супералгебра Ли, элементы которой имеют вид

$$
\sum f_{i}(x) \frac{\partial}{\partial x_{i}}
$$

Мы рассматриваем внешний дифференциал как супердифференцирование супералгебры внешних форм. Поэтому для любого нечетного $x$ дифференциал $d x$ четен, и можно рассматривать не только многочлены относительно $d x$. Гладкие или аналитические функции от дифференциалов $d x$ называются псевдодифференциальными формами на супермногообразиях с координатами $x$ [57]. Такие формы используются при интерпретации $\mathfrak{h}_{\lambda}(2 \mid 2)$.

П.1.1. Два типа общих линейных супералгебр. Обшая линейная супералгебра Ли всех суперматриц формата $\mathrm{Par}$ (упорядоченный набор четностей базисных векторов или просто суперразмерность) обозначается символом $\mathfrak{g l}(\mathrm{Par})$. Любая матрица из $\mathfrak{g l}(\mathrm{Par})$ может быть представлена в виде суммы ее четной и нечетной частей; в стандартном формате, используя блочные обозначения, имеем

$$
\left(\begin{array}{ll}
A & B \\
C & D
\end{array}\right)=\left(\begin{array}{ll}
A & 0 \\
0 & D
\end{array}\right)+\left(\begin{array}{cc}
0 & B \\
C & 0
\end{array}\right)
$$


где

$$
p\left(\left(\begin{array}{cc}
A & 0 \\
0 & D
\end{array}\right)\right)=\overline{0}, \quad p\left(\left(\begin{array}{cc}
0 & B \\
C & 0
\end{array}\right)\right)=\overline{1} .
$$

Суперслед $\mathrm{str}$ - это отображение $\mathfrak{g l}(\mathrm{Par}) \rightarrow \mathbb{C}, \quad\left(A_{i j}\right) \mapsto \sum(-1)^{p_{i}} A_{i i}$. Поскольку $\operatorname{str}[x, y]=0$, подсуперпространство суперматриц с нулевым суперследом образует сnеииальную линейную супералгебру Ли $\mathfrak{s l}(\mathrm{Par})$.

Другой аналог $\mathfrak{g l}(n)$ называется странной супералгеброй Ли и определяется как супералгебра Ли q $(n)$, сохраняющая комплексную структуру, заданную некоторым нечетным оператором $J$, т.е. $\mathfrak{q}(n)$ является централизатором $C(J)$ оператора $J$ :

$$
\mathfrak{q}(n)=C(J)=\{X \in \mathfrak{g l}(n \mid n) \mid[X, J]=0\},
$$

где $J^{2}=-\mathrm{id}$. Ясно, что, изменяя базис, мы можем привести $J$ к виду

$$
J_{2 n}=\left(\begin{array}{cc}
0 & 1_{n} \\
-1 & 0
\end{array}\right)
$$

В стандартном формате имеем

$$
\mathfrak{q}(n)=\left\{\left(\begin{array}{cc}
A & B \\
B & A
\end{array}\right)\right\}
$$

На $\mathfrak{q}(n)$ странньй след определяется следующим образом:

$$
\mathrm{qtr}:\left(\begin{array}{cc}
A & B \\
B & A
\end{array}\right) \mapsto \operatorname{tr} B
$$

Обозначим символом $\mathfrak{s q}(n)$ супералгебру Ли матриц $c$ нулевым странным следом.

Заметим, что тождественные представления $\mathfrak{q}$ и $\mathfrak{s q}$ в $V$, хотя и неприводимы в суперсмысле, приводимы в неградуированном смысле. Чтобы в этом убедиться, достаточно взять однородные относительно четности и линейно независимые векторы $v_{1}, \ldots, v_{n}$ из $V$. Тогда $\operatorname{Span}\left(v_{1}+J\left(v_{1}\right), \ldots, v_{n}+J\left(v_{n}\right)\right)$ есть инвариантное подпространство пространства $V$, не являющееся подсуперпространством.

Представление называется неприводимым общего типа или $G$-типа, если в нем нет нетривиального инвариантного подпространства. Неприводимое представление называется неприводимым $Q$-типа ( $Q$ указывает на обшую странную (queer) супералгебру Ли), если у него нет инвариантного подсуперпространства, но есть нетривиальное инвариантное подпространство.

П.1.2. Два типа супералгебр Ли, сохраняющих билинейные формы. Каждому линейному оператору $F$ суперпространств соответствует дуальный оператор $F^{*}$ дуальных суперпространств; если $A$ - суперматрица, соответствуюшая оператору $F$ в некотором базисе формата $\mathrm{Par}$, то оператору $F^{*}$ соответствует супертранспонированная матрица $A^{\text {st }}$, определяемая формулой

$$
\left(A^{\mathrm{st}}\right)_{i j}=(-1)^{\left(p_{i}+p_{j}\right)\left(p_{i}+p(A)\right)} A_{j i}
$$


Суперматрицы $X \in \mathfrak{g l}(\mathrm{Par})$ такие, что

$$
X^{\text {st }} B+(-1)^{p(X) p(B)} B X=0
$$

для некоторой однородной матрицы $B \in \mathfrak{g l}(\mathrm{Par})$, образуют супералгебру Ли $\mathfrak{a u t}(B)$, coхраняющую билинейную форму на $V$ с матрищей $B$.

Напомним, что однородная форма $\omega$ суперсимметрична, если ее матрица $B$ удовлетворяет условию $B^{u}=B$, где

$$
B^{u}=\left(\begin{array}{cc}
R^{\mathrm{t}} & (-1)^{p(B)} T^{\mathrm{t}} \\
(-1)^{p(B)} S^{\mathrm{t}} & -U^{\mathrm{t}}
\end{array}\right), \quad B=\left(\begin{array}{cc}
R & S \\
T & U
\end{array}\right) .
$$

Аналогично матрица $B$ суперкососимметрична, если $B^{u}=-B$. Таким образом, мы видим, что переворачивание билинейных форм $u: \operatorname{Bil}(V, W) \rightarrow \operatorname{Bil}(W, V)$, которое в чисто четном случае сводится к транспонированию матриц, является новой операцией.

Наиболее распространенные канонические формы невырожденных суперсимметрических форм - это те формы, суперматрицы которых в стандартном формате суть следующие канонические суперматрицы $B_{\mathrm{ev}}$ или $B_{\mathrm{ev}}^{\prime}$ :

$$
B_{\mathrm{ev}}(m \mid 2 n)=\left(\begin{array}{cc}
1_{m} & 0 \\
0 & J_{2 n}
\end{array}\right), \quad J_{2 n}=\left(\begin{array}{cc}
0 & 1_{n} \\
-1_{n} & 0
\end{array}\right),
$$

или

$$
B_{\mathrm{ev}}^{\prime}(m \mid 2 n)=\left(\begin{array}{cc}
\operatorname{antidiag}(1, \ldots, 1) & 0 \\
0 & J_{2 n}
\end{array}\right) .
$$

Обычно $\mathfrak{a u t}\left(B_{\mathrm{ev}}(m \mid 2 n)\right)$ обозначается как $\mathfrak{o s p}(m \mid 2 n)$ или, более точно, osp $\mathfrak{p}^{s y}(m \mid 2 n)$. Заметим, что при переходе от $V$ к П $(V)$ суперсимметрические формы преврашаются в суперкососимметрические, сохраняемые “симплектико-ортогональной" супералгеброй Ли $\mathfrak{s p o}(2 n \mid m)$ или, точнее, osp ${ }^{s k}(m \mid 2 n)$, которая изоморфна osp ${ }^{s y}(m \mid 2 n)$, но имеет иную матричную реализацию, однако мы не используем обозначение $\mathfrak{p} \mathfrak{p}(2 n \mid m)$, чтобы не спутать ее со специальной пуассоновой супералгеброй.

В стандартном формате матричные реализации вышеупомянутых супералгебр суть

$$
\mathfrak{o} \mathfrak{s p}(m \mid 2 n)=\left\{\left(\begin{array}{ccc}
E & Y & X^{\mathrm{t}} \\
X & A & B \\
-Y^{\mathrm{t}} & C & -A^{\mathrm{t}}
\end{array}\right)\right\}, \quad \mathfrak{o s p}^{s k}(m \mid 2 n)=\left\{\left(\begin{array}{ccc}
A & B & X \\
C & -A^{\mathrm{t}} & Y^{\mathrm{t}} \\
Y & -X^{\mathrm{t}} & E
\end{array}\right)\right\}
$$

где

$$
\left(\begin{array}{cc}
A & B \\
C & -A^{\mathrm{t}}
\end{array}\right) \in \mathfrak{s p}(2 n)
$$

$E \in \mathfrak{o}(m)$ и $\mathrm{t}-$ знак обычного транспонирования.

Невырожденная суперсимметрическая нечетная билинейная форма $B_{\text {odd }}(n \mid n)$ может быть приведена к каноническому виду с матрицей, которая в стандартном формате равна $J_{2 n}$. Канонический вид суперкососимметрической нечетной невырожденной формы в стандартном формате есть

$$
\Pi_{2 n}=\left(\begin{array}{cc}
0 & 1_{n} \\
1_{n} & 0
\end{array}\right) .
$$


Обрашаем внимание на то, что у нас нет здесь ошибки в знаке: матрица с минусом отвечает суперсимметрической форме, а без минуса - суперкососимметрической!

Обычно $\mathfrak{a u t}\left(B_{\text {odd }}(\mathrm{Par})\right)$ обозначается символом $\mathfrak{p}(\mathrm{Par})$. Переход от $V$ к П( $\left.V\right)$ устанавливает изоморфизм $\mathfrak{p e}^{s y}(\mathrm{Par}) \cong \mathfrak{p e}^{s k}(\mathrm{Par})$. По предложению Вейля эта супералгебра Ли называется периплектической. В стандартном формате матричные реализации вышеупомянутых супералгебр суть

$$
\begin{aligned}
& \mathfrak{p e}^{s y}(n)=\left\{\left(\begin{array}{cc}
A & B \\
C & -A^{\mathrm{t}}
\end{array}\right), \text { где } B=-B^{\mathrm{t}}, \quad C=C^{\mathrm{t}}\right\}, \\
& \mathfrak{p e}^{s k}(n)=\left\{\left(\begin{array}{cc}
A & B \\
C & -A^{\mathrm{t}}
\end{array}\right), \text { где } B=B^{\mathrm{t}}, \quad C=-C^{\mathrm{t}}\right\} .
\end{aligned}
$$

(Заметим, что хотя супералгебры Ли osp $\mathfrak{p}^{s y}(m \mid 2 n)$ и osp $\mathfrak{p}^{s k}(2 n \mid m)$ изоморфны, так же как $\mathfrak{p} \mathfrak{e}^{s y}(n)$ и $\mathfrak{p e}^{s k}(n)$, различие между ними иногда имеет решаюшее значение, см. [39] и замечание в п. П.5.2.)

Специальной периплектической супералгеброй называется $\mathfrak{s p e}(n)=\{X \in \mathfrak{p} \mathfrak{e}(n) \mid$ $\operatorname{str} X=0\}$. Особый интерес, как главная линейная часть алгебры $\mathfrak{b}_{\lambda}(n)$, представляет также $\mathfrak{s p e}(n)_{a, b}=\mathfrak{s p e}(n) \boxplus \mathbb{C}(a z+b d)$, где $z=1_{2 n}$ и $d=\operatorname{diag}\left(1_{n},-1_{n}\right)$, а $\boxplus-$ знак полупрямой суммы (идеал слева).

\section{П.2. Векторные супералгебры Ли. Стандартная реализация}

Элементы алгебры Ли $\mathcal{L}=\mathfrak{d e r} \mathbb{C}[u]$ интерпретируются как векторные поля. Алгебра Ли $\mathcal{L}$ имеет только одну максимальную подалгебру $\mathcal{L}_{0}$ конечной коразмерности (состоящую из полей, обрашаюшихся в нуль в начале координат). Подалгебра $\mathcal{L}_{0}$ определяет фильтрацию в $\mathcal{L}:$

$$
\mathcal{L}_{-1}=\mathcal{L} \quad \text { и } \quad \mathcal{L}_{i}=\left\{D \in \mathcal{L}_{i-1} \mid[D, \mathcal{L}] \subset \mathcal{L}_{i-1}\right\} \text { при } i \geqslant 1 .
$$

Ассоциированная градуированная алгебра Ли $L=\bigoplus_{i \geqslant-1} L_{i}$, где $L_{i}=\mathcal{L}_{i} / \mathcal{L}_{i+1}$, состоит из векторных полей с полиномиальными коэффициентами.

Суперизация. Пусть $\mathcal{L}=\mathfrak{d e r} \mathbb{C}[u, \xi]$. Предположим, что $\mathcal{L}_{0} \subset \mathcal{L}$ - максимальная подалгебра конечной коразмерности, не содержашая идеалов $\mathcal{L}$. Пусть $\mathcal{L}_{-1}$ - минимальное подпространство в $\mathcal{L}$, содержашее $\mathcal{L}_{0}$, отличное от $\mathcal{L}_{0}$ и $\mathcal{L}_{0}$-инвариантное. Фильтрачия Вейсфеллера супералгебры $\mathcal{L}$ определяется при $i \geqslant 1$ формулами

$$
\mathcal{L}_{-i-1}=\left[\mathcal{L}_{-1}, \mathcal{L}_{-i}\right]+\mathcal{L}_{-i}, \quad \mathcal{L}_{i}=\left\{D \in \mathcal{L}_{i-1} \mid\left[D, \mathcal{L}_{-1}\right] \subset \mathcal{L}_{i-1}\right\}
$$

Поскольку коразмерность $\mathcal{L}_{0}$ конечна, фильтрация имеет вид

$$
\mathcal{L}=\mathcal{L}_{-d} \supset \cdots \supset \mathcal{L}_{0} \supset \cdots
$$

для некоторой глубины $d$. Рассматривая подпространства (П.1) в качестве базы некоторой топологии, мы можем пополнить градуированные $L$ или фильтрованные супералгебры Ли $\mathcal{L}$; элементами пополнения являются векторные поля, коэффищиенты которых - формальные степенные ряды. Хотя структуру градуированных алгебр легче описывать, пополненные супералгебры Ли часто необходимы в приложениях. 
В отличие от алгебр Ли простые векторные супералгебры обладают несколькими неизоморфными максимальными подалгебрами конечной коразмерности (см. п. П.7).

1. Общие алгебрь Ли. Пусть $x=\left(u_{1}, \ldots, u_{n}, \theta_{1}, \ldots, \theta_{m}\right)$, где $u_{i}$ и $\theta_{j}-$ соответственно четные и нечетные переменные. Общей векторной супералгеброй Ли называется $\mathfrak{v e c t}(n \mid m)=\mathfrak{d e r} \mathbb{C}[x]$.

2. Специальные алгебры. Дивергениией поля

$$
D=\sum_{i} f_{i} \frac{\partial}{\partial u_{i}}+\sum_{j} g_{j} \frac{\partial}{\partial \theta_{j}}
$$

называется функция (в нашем случае многочлен или ряд)

$$
\operatorname{div} D=\sum_{i} \frac{\partial f_{i}}{\partial u_{i}}+\sum_{j}(-1)^{p\left(g_{j}\right)} \frac{\partial g_{i}}{\partial \theta_{j}} .
$$

Супералгебра Ли $\mathfrak{s v e c t}(n \mid m)=\{D \in \mathfrak{v e c t}(n \mid m) \mid \operatorname{div} D=0\}$ назьвается специальной или бездивергентной векторной супералгеброй.

Ясно, что можно описать $\mathfrak{s v e c t}(n \mid m)$ как $\left\{D \in \mathfrak{v e c t}(n \mid m) \mid L_{D} \operatorname{vol}_{x}=0\right\}$, где $\operatorname{vol}_{x}-$ элемент объема с постоянными коэффициентами в координатах $x$ (см. п. П.6), $L_{D}$ - производная Ли вдоль $D$.

Супералгебра Ли $\mathfrak{s v e c t}_{\lambda}(0 \mid m)=\left\{D \in \mathfrak{v e c t}(0 \mid m) \mid \operatorname{div}\left(1+\lambda \theta_{1} \ldots \theta_{m}\right) D=0\right\}$, где $p(\lambda) \equiv m(\bmod 2),-$ деформация svect $(0 \mid m)$ - называется деформированной специальной векторной супералгеброй. Ясно, что $\mathfrak{s v e c t}_{\lambda}(0 \mid m) \cong \mathfrak{s v e c t}_{\mu}(0 \mid m)$ при $\lambda \mu \neq 0$. Поэтому мы будем для краткости обозначать эти деформации при $\lambda \neq 0$ символом $\widetilde{\mathfrak{s v e c t}}(0 \mid m)$. Заметим, что для нечетных $m$ параметр деформации $\lambda$ нечетен.

3. Алгебры, сохраняющие уравнения Пфаффа и дифференциальные 2-формы. Положим $u=\left(t, p_{1}, \ldots, p_{n}, q_{1}, \ldots, q_{n}\right)$,

$$
\tilde{\alpha}_{1}=d t+\sum_{1 \leqslant i \leqslant n}\left(p_{i} d q_{i}-q_{i} d p_{i}\right)+\sum_{1 \leqslant j \leqslant m} \theta_{j} d \theta_{j}, \quad \widetilde{\omega}_{0}=\tilde{d} \alpha_{1} .
$$

Форма $\tilde{\alpha}_{1}$ называется контактной, форма $\widetilde{\omega}_{0}$ называется симплектической. Иногда более удобно переобозначить переменные $\theta$ и положить

$$
\begin{gathered}
\xi_{j}=\frac{1}{\sqrt{2}}\left(\theta_{j}-i \theta_{r+j}\right), \quad \eta_{j}=\frac{1}{\sqrt{2}}\left(\theta_{j}+i \theta_{r+j}\right) \\
\text { при } \left.\quad j \leqslant r=[m / 2] \quad \text { (3десь } i^{2}=-1\right), \quad \theta=\theta_{2 r+1},
\end{gathered}
$$

а вместо $\tilde{\alpha}_{1}$ и $\widetilde{\omega}_{0}$ использовать $\alpha_{1}$ и $\omega_{0}=d \alpha_{1}$, где

$$
\alpha_{1}=d t+\sum_{1 \leqslant i \leqslant n}\left(p_{i} d q_{i}-q_{i} d p_{i}\right)+\sum_{1 \leqslant j \leqslant r}\left(\xi_{j} d \eta_{j}+\eta_{j} d \xi_{j}\right)+ \begin{cases}0 & \text { при } m=2 r, \\ \theta d \theta & \text { при } m=2 r+1 .\end{cases}
$$

Супералгебра Ли, которая сохраняет уравнение Пфаффа $\alpha_{1}=0$, т.е. супералгебра

$$
\begin{aligned}
& \mathfrak{k}(2 n+1 \mid m)= \\
& \quad=\left\{D \in \mathfrak{v e c t}(2 n+1 \mid m) \mid L_{D} \alpha_{1}=f_{D} \alpha_{1} \text { для некоторого } f_{D} \in \mathbb{C}[t, p, q, \theta]\right\},
\end{aligned}
$$


называется контактной супералгеброй. Супералгебра Ли

$$
\mathfrak{p o}(2 n \mid m)=\left\{D \in \mathfrak{k}(2 n+1 \mid m) \mid L_{D} \alpha_{1}=0\right\}
$$

называется супералгеброй Пуассона.(С геометрической точки зрения супералгебра Пуассона - это супералгебра Ли, которая сохраняет связность с формой $\alpha$ в линейном расслоении над симплектическим супермногообразием с симплектической формой $d \alpha$.

Положим $q=\left(q_{1}, \ldots, q_{n}\right)$ и пусть $\theta=\left(\xi_{1}, \ldots, \xi_{n} ; \tau\right)$. Положим

$$
\alpha_{0}=d \tau+\sum_{i}\left(\xi_{i} d q_{i}+q_{i} d \xi_{i}\right), \quad \omega_{1}=d \alpha_{0}
$$

Эти формы называются нечетно-контактной и периплектической, соответственно. (Заметим, что нечетно-контактная форма $\alpha_{0}$ четна; просто она является "нечетным" аналогом “обычной” контактной структуры.)

Супералгебра Ли, которая сохраняет уравнение Пфаффа $\alpha_{0}=0$, т.е. супералгебра

$$
\mathfrak{m}(n)=\left\{D \in \mathfrak{v e c t}(n \mid n+1) \mid L_{D} \alpha_{0}=f_{D} \cdot \alpha_{0} \text { для некоторого } f_{D} \in \mathbb{C}[q, \xi, \tau]\right\},
$$

называется нечетно-контактной супералгеброй.

Супералгебра Ли

$$
\mathfrak{b}(n)=\left\{D \in \mathfrak{m}(n) \mid L_{D} \alpha_{0}=0\right\}
$$

называется супералгеброй Бюттен. (С геометрической точки зрения супералгебра Бюттен - это супералгебра Ли, которая сохраняет связность с формой $\alpha_{1}$ в линейном расслоении ранга $\varepsilon$ над периплектическим супермногообразием с периплектической формой $d \alpha_{0}$.)

Супералгебры Ли

$$
\mathfrak{s m}(n)=\{D \in \mathfrak{m}(n) \mid \operatorname{div} D=0\}, \quad \mathfrak{s b}(n)=\{D \in \mathfrak{b}(n) \mid \operatorname{div} D=0\}
$$

называются спещиальной нечетно-контактной и специальной супералгеброй Бюттен, соответственно.

ЗАмЕчаниЕ. Связь этих супералгебр Ли с конечномерной геометрией следующая. Ясно, что ker $\alpha_{1}=\operatorname{ker} \tilde{\alpha}_{1}$. Ограничение $\widetilde{\omega}_{0}$ на ker $\tilde{\alpha}_{1}$ является ортосимплектической формой $B_{\mathrm{ev}}(m \mid 2 n)$, ограничение $\omega_{0}$ на $\operatorname{ker} \alpha_{1}$ есть $B_{\mathrm{ev}}^{\prime}(m \mid 2 n)$. Аналогично ограничение $\omega_{1}$ на $\operatorname{ker} \alpha_{0}$ есть $B_{\text {odd }}(n \mid n)$.

\section{П.3. Производящие функции}

Для лаконичного описания супералгебр $\mathfrak{k}, \mathfrak{m}$ и их подалгебр служат производяшие функции. 
Нечетная форма $\alpha_{1}$. Каждому контактному векторному полю $D \in \mathfrak{k}(2 n+1 \mid m)$ можно сопоставить некоторую функцию $f \in \mathbb{C}[t, p, q, \theta]$, называемую производяшей функцией этого векторного поля. Однако удобные формулы задают не само отображение $\mathfrak{k}(2 n+1 \mid m) \rightarrow \mathbb{C}[t, p, q, \theta]$, а его обратное: $\mathbb{C}[t, p, q, \theta] \rightarrow \mathfrak{k}(2 n+1 \mid m), f \mapsto K_{f}$. Для $f \in \mathbb{C}[t, p, q, \theta]$ положим

$$
K_{f}=(2-E)(f) \frac{\partial}{\partial t}-H_{f}+\frac{\partial f}{\partial t} E
$$

где $2(f)=2 \cdot f$,

$$
E=\sum_{i} y_{i} \frac{\partial}{\partial y_{i}}
$$

- это оператор Эйлера, который вычисляет степень относительно переменных $y$ (здесь $y_{i}$ - все координаты, за исключением $t$ ), а $H_{f}$ задается формулой

$$
H_{f}=\sum_{i \leqslant n}\left(\frac{\partial f}{\partial p_{i}} \frac{\partial}{\partial q_{i}}-\frac{\partial f}{\partial q_{i}} \frac{\partial}{\partial p_{i}}\right)-(-1)^{p(f)}\left(\sum_{j \leqslant m} \frac{\partial f}{\partial \theta_{j}} \frac{\partial}{\partial \theta_{j}}\right) .
$$

Выбор формы $\alpha_{1}$ вместо $\tilde{\alpha}_{1}$ меняет только вид $H_{f}$, который мы приводим для $m=$ $2 k+1$ :

$$
H_{f}=\sum_{i \leqslant n}\left(\frac{\partial f}{\partial p_{i}} \frac{\partial}{\partial q_{i}}-\frac{\partial f}{\partial q_{i}} \frac{\partial}{\partial p_{i}}\right)-(-1)^{p(f)}\left(\sum_{j \leqslant k}\left(\frac{\partial f}{\partial \xi_{j}} \frac{\partial}{\partial \eta_{j}}+\frac{\partial f}{\partial \eta_{j}} \frac{\partial}{\partial \xi_{j}}\right)+\frac{\partial f}{\partial \theta} \frac{\partial}{\partial \theta}\right) .
$$

Четная форма $\alpha_{0}$. Аналогично предыдушему каждому нечетно-контактному векторному полю $D \in \mathfrak{m}(n)$ можно сопоставить производящую функцию $f \in \mathbb{C}[q, \xi, \tau]$. При этом удобные формулы задают обратное отображение: $\mathbb{C}[q, \xi, \tau] \rightarrow \mathfrak{m}(n), f \mapsto M_{f}$. Для $f \in \mathbb{C}[q, \xi, \tau]$ положим

$$
M_{f}=(2-E)(f) \frac{\partial}{\partial \tau}-L e_{f}-(-1)^{p(f)} \frac{\partial f}{\partial \tau} E,
$$

где $E$ определяется так же, как в (П.4), и

$$
L e_{f}=\sum_{i \leqslant n}\left(\frac{\partial f}{\partial q_{i}} \frac{\partial}{\partial \xi_{i}}+(-1)^{p(f)} \frac{\partial f}{\partial \xi_{i}} \frac{\partial}{\partial q_{i}}\right) .
$$

Поскольку

$$
\begin{aligned}
& L_{K_{f}}\left(\alpha_{1}\right)=2 \frac{\partial f}{\partial t} \alpha_{1}=K_{1}(f) \alpha_{1}, \\
& L_{M_{f}}\left(\alpha_{0}\right)=-(-1)^{p(f)} 2 \frac{\partial f}{\partial \tau} \alpha_{0}=-(-1)^{p(f)} M_{1}(f) \alpha_{0},
\end{aligned}
$$

то получается, что $K_{f} \in \mathfrak{k}(2 n+1 \mid m)$ и $M_{f} \in \mathfrak{m}(n)$. Заметим, что

$$
p\left(L e_{f}\right)=p\left(M_{f}\right)=p(f)+\overline{1} .
$$


(Супер)коммутаторам векторных полей $\left[K_{f}, K_{g}\right]$ или $\left[M_{f}, M_{g}\right]$ соответствуют контактные скобки производящих функций

$$
\left[K_{f}, K_{g}\right]=K_{\{f, g\}_{k . b .}}, \quad\left[M_{f}, M_{g}\right]=M_{\{f, g\}_{m . b .}} .
$$

Приведем явные формулы для контактных скобок. Сначала определим скобки Пуассона и Бюттен.

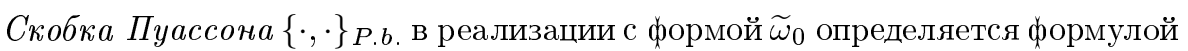

$$
\{f, g\}_{P . b .}=\sum_{i \leqslant n}\left(\frac{\partial f}{\partial p_{i}} \frac{\partial g}{\partial q_{i}}-\frac{\partial f}{\partial q_{i}} \frac{\partial g}{\partial p_{i}}\right)-(-1)^{p(f)} \sum_{j \leqslant m} \frac{\partial f}{\partial \theta_{j}} \frac{\partial g}{\partial \theta_{j}},
$$

а в реализации с формой $\omega_{0}$ при $m=2 k+1$ определяется формулой

$$
\{f, g\}_{P . b .}=\sum_{i \leqslant n}\left(\frac{\partial f}{\partial p_{i}} \frac{\partial g}{\partial q_{i}}-\frac{\partial f}{\partial q_{i}} \frac{\partial g}{\partial p_{i}}\right)-(-1)^{p(f)}\left[\sum_{j \leqslant m}\left(\frac{\partial f}{\partial \xi_{j}} \frac{\partial g}{\partial \eta_{j}}+\frac{\partial f}{\partial \eta_{j}} \frac{\partial g}{\partial \xi_{j}}\right)+\frac{\partial f}{\partial \theta} \frac{\partial g}{\partial \theta}\right] .
$$

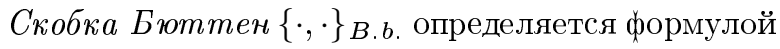

$$
\{f, g\}_{\text {B.b. }}=\sum_{i \leqslant n}\left(\frac{\partial f}{\partial q_{i}} \frac{\partial g}{\partial \xi_{i}}+(-1)^{p(f)} \frac{\partial f}{\partial \xi_{i}} \frac{\partial g}{\partial q_{i}}\right) .
$$

В терминах скобок Пуассона и Бюттен контактные скобки имеют вид

$$
\begin{aligned}
\{f, g\}_{k . b .} & =(2-E)(f) \frac{\partial g}{\partial t}-\frac{\partial f}{\partial t}(2-E)(g)-\{f, g\}_{P . b .} \\
\{f, g\}_{m . b .} & =(2-E)(f) \frac{\partial g}{\partial \tau}+(-1)^{p(f)} \frac{\partial f}{\partial \tau}(2-E)(g)-\{f, g\}_{B . b .} .
\end{aligned}
$$

Из (П.8) видно, что отображение $f \mapsto K_{f}$ устанавливает изоморфизм между подпространством $\mathbb{C}[p, q, \theta]$ функций, не зависящих от $t$, и супералгеброй Ли $\mathfrak{p o}(2 n \mid m)$, а отображение $f \mapsto M_{f}$ - между подпространством $\mathbb{C}[q, \xi]$ функций, не зависящих от $\tau$, и супералгеброй Бюттен $\mathfrak{b}(n)$. При этом контактная скобка на $\mathbb{C}[p, q, \theta]$ совпадает с точностью до множителя со скобкой Пуассона, а нечетно-контактная скобка на $\mathbb{C}[q, \xi]-$ со скобкой Бюттен.

Супералгебра Ли гамильтоновых полей (или гамильтонова супералгебра) и ее специальная подалгебра (определенная только при $n=0$ ) суть

$$
\mathfrak{h}(2 n \mid m)=\left\{D \in \mathfrak{v e c t}(2 n \mid m) \mid L_{D} \omega_{0}=0\right\}
$$

и

$$
\mathfrak{s h}(m)=\left\{H_{f} \in \mathfrak{h}(0 \mid m) \mid \int f \operatorname{vol}_{\theta}=0\right\} .
$$

Это прямые аналоги гамильтоновой алгебры Ли $\mathfrak{h}(2 n)$.

Непрямыми аналогами $\mathfrak{h}(2 n)$, отражающими особые возможности именно суперпространств, являются супералгебра Ли векторных полей $\mathfrak{l}(n)$, введенная в работе [2], и ее специальная подалгебра

$$
\mathfrak{l e}(n)=\left\{D \in \mathfrak{v e c t}(n \mid n) \mid L_{D} \omega_{1}=0\right\} \quad \text { и } \quad \mathfrak{s l} \mathfrak{e}(n)=\{D \in \mathfrak{l e}(n) \mid \operatorname{div} D=0\} .
$$

Нетрудно доказать следуюшие изоморфизмы суперпространств:

$$
\begin{array}{ll}
\mathfrak{k}(2 n+1 \mid m) \cong \operatorname{Span}\left(K_{f} \mid f \in \mathbb{C}[t, p, q, \theta]\right), & \mathfrak{h}(2 n \mid m) \cong \operatorname{Span}\left(H_{f} \mid f \in \mathbb{C}[p, q, \theta]\right), \\
\mathfrak{m}(n) \cong \operatorname{Span}\left(M_{f} \mid f \in \mathbb{C}[\tau, q, \xi]\right), & \mathfrak{l}(n) \cong \operatorname{Span}\left(L e_{f} \mid f \in \mathbb{C}[q, \xi]\right) .
\end{array}
$$




\section{П.4. Бездивергентные подалгебры}

Поскольку

$$
\operatorname{div} K_{f}=(2 n+2-m) K_{1}(f),
$$

то бездивергентная подалгебра контактной супералгебры Ли либо совпадает с ней (при $m=2 n+2)$, либо изоморфна супералгебре Пуассона. Для нечетно-контактной серии ситуация более интересна: бездивергентная подалгебра проста при $n>1$ и $n \neq 3$. Поскольку

$$
\operatorname{div} M_{f}=(-1)^{p(f)} 2\left((1-E) \frac{\partial f}{\partial \tau}-\sum_{i \leqslant n} \frac{\partial^{2} f}{\partial q_{i} \partial \xi_{i}}\right),
$$

получаем следуюшее явное ее описание:

$$
\mathfrak{s m}(n)=\operatorname{Span}\left(M_{f} \in \mathfrak{m}(n) \mid(1-E) \frac{\partial f}{\partial \tau}=\sum_{i \leqslant n} \frac{\partial^{2} f}{\partial q_{i} \partial \xi_{i}}\right) .
$$

В частности,

$$
\operatorname{div} L e_{f}=(-1)^{p(f)} 2 \sum_{i \leqslant n} \frac{\partial^{2} f}{\partial q_{i} \partial \xi_{i}}
$$

Нечетный аналог лапласиана, т.е. оператор

$$
\Delta=\sum_{i \leqslant n} \frac{\partial^{2}}{\partial q_{i} \partial \xi_{i}}
$$

на периплектическом супермногообразии, называется в работах по физике БРСТ-оператором (см., например, [30]). Векторные поля из $\mathfrak{s l e}(n)$ порождаются гармоническими функциями, т.е. такими, что $\Delta f=0$.

\section{П.5. Картановские продолжения}

Чтобы определить $\mathfrak{b}_{\lambda}(n)$, а также некоторые связанные с ней алгебры, нам понадобится понятие картановского продолжения. Пусть $\mathfrak{g}$ - алгебра Ли, $V$ - некоторый $\mathfrak{g}$-модуль, $S^{i}$ - оператор $i$-й симметрической степени. Положим $\mathfrak{g}_{-1}=V, \mathfrak{g}_{0}=\mathfrak{g}$ и при $i>0$ определим $i$-е картановское продолжение пары $\left(\mathfrak{g}_{-1}, \mathfrak{g}_{0}\right)$ как

$$
\begin{aligned}
\mathfrak{g}_{i} & =\left\{X \in \operatorname{Hom}\left(\mathfrak{g}_{-1}, \mathfrak{g}_{i-1}\right) \mid X\left(v_{0}\right)\left(v_{1}, \ldots, v_{i}\right)=\right. \\
& \left.=X\left(v_{1}\right)\left(v_{0}, \ldots, v_{i}\right) \quad \text { для любых } v_{0}, v_{1}, \ldots, v_{i} \in \mathfrak{g}_{-1}\right\}= \\
& =\left(S^{i}\left(\mathfrak{g}_{-1}^{*}\right) \otimes \mathfrak{g}_{0}\right) \cap\left(S^{i+1}\left(\mathfrak{g}_{-1}^{*}\right) \otimes \mathfrak{g}_{-1}\right)
\end{aligned}
$$

(мы здесь трактуем $\mathfrak{g}_{0}$ как подпространство в $\mathfrak{g}_{-1}^{*} \otimes \mathfrak{g}_{-1}$, поэтому пересечение корректно определено).

Картановское продолжение пары $(V, \mathfrak{g})$ - это $\left(\mathfrak{g}_{-1}, \mathfrak{g}_{0}\right)_{*}=\bigoplus_{i \geqslant-1} \mathfrak{g}_{i}$.

Предположим, что $\mathfrak{g}_{-1}-$ точный $\mathfrak{g}_{0}$-модуль (т.е. $\mathfrak{g}_{0}$ действует с нулевым ядром). Тогда, конечно,

$$
\left(\mathfrak{g}_{-1}, \mathfrak{g}_{0}\right)_{*} \subset \mathfrak{v e c t}(n)=\mathfrak{d e r} \mathbb{C}\left[x_{1}, \ldots, x_{n}\right]
$$


где

$$
n=\operatorname{dim} \mathfrak{g}_{-1} \quad \text { и } \quad \mathfrak{g}_{i}=\left\{D \in \operatorname{vect}(n) \mid \operatorname{deg} D=i,[D, X] \in \mathfrak{g}_{i-1} \quad \text { для любых } X \in \mathfrak{g}_{-1}\right\} .
$$

Нетрудно проверить что структура алгебры Ли на $\mathfrak{v e c t}(n)$ индуцирует структуру алгебры Ли на $\left(\mathfrak{g}_{-1}, \mathfrak{g}_{0}\right)_{*}$.

Из четырех простых векторных алгебр Ли три являются картановскими продолжениями: $\mathfrak{v e c t}(n)=(\mathrm{id}, \mathfrak{g l}(n))_{*}, \mathfrak{s v e c t}(n)=(\mathrm{id}, \mathfrak{s l}(n))_{*}$ и $\mathfrak{h}(2 n)=(\mathrm{id}, \mathfrak{s p}(n))_{*}$, а четвертая $-\mathfrak{k}(2 n+1)-$ результат следуюшей чуть более обшей процедуры.

П.5.1. Обобщение картановского продолжения. Пусть

$$
\mathfrak{g}_{-}=\bigoplus_{-d \leqslant i \leqslant-1} \mathfrak{g}_{i}
$$

- нильпотентная $\mathbb{Z}$-градуированная алгебра Ли, $\mathfrak{g}_{0} \subset \mathfrak{d e r}_{0} \mathfrak{g}-$ подалгебра в алгебре Ли дифференцирований, сохраняющих $\mathbb{Z}$-градуировку. При $i>0$ определим $i$-е продолжение пары $\left(\mathfrak{g}_{-}, \mathfrak{g}_{0}\right)$ как

$$
\mathfrak{g}_{i}=\left(\left(S^{\bullet}\left(\mathfrak{g}_{-}^{*}\right) \otimes \mathfrak{g}_{0}\right) \cap\left(S^{\bullet}\left(\mathfrak{g}_{-}^{*}\right) \otimes \mathfrak{g}_{-}\right)\right)_{i}
$$

где индекс $i$ в правой части обозначает компоненту степени $i$.

Определим $\left(\mathfrak{g}_{-}, \mathfrak{g}_{0}\right)_{*}$ как $\bigoplus_{i \geqslant-d} \mathfrak{g}_{i}$. Тогда нетрудно проверить, что $\left(\mathfrak{g}_{-}, \mathfrak{g}_{0}\right)_{*}-$ алгебра Ли.

Выясним, что в этих терминах представляет собой алгебра Ли контактных векторных полей. Обозначим символом $\mathfrak{h} \mathfrak{e}(2 n)$ алгебру Гейзенберга. Ее пространством является $W \oplus \mathbb{C} \cdot z$, где $W$ есть $2 n$-мерное пространство с невырожденной кососимметрической билинейной формой $B$. Скобка в $\mathfrak{h} \mathfrak{e i}(2 n)$ определяется следующим образом:

Элемент $z$ четен и лежит в центре; $[v, w]=B(v, w) \cdot z$ для любых $v, w \in W$.

Теперь понятно, что $\mathfrak{k}(2 n+1) \cong(\mathfrak{h} \mathfrak{e i}(2 n), \mathfrak{c s p}(2 n))_{*}$.

П.5.2. Супералгебры векторных полей как картановские продолжения. Применяя к рассуждениям п. П.5 и П.5.1 правило знаков, мы получаем

$$
\begin{aligned}
\mathfrak{v e c t}(m \mid n)= & (\mathrm{id}, \mathfrak{g l}(m \mid n))_{*}, \quad \mathfrak{s v e c t}(m \mid n)=(\mathrm{id}, \mathfrak{s l}(m \mid n))_{*}, \\
& \mathfrak{h}(2 m \mid n)=\left(\mathrm{id}, \mathfrak{o s p}^{s k}(m \mid 2 n)\right)_{*} \\
\mathfrak{l e}(n)= & \left(\mathrm{id}, \mathfrak{p} \mathfrak{e}^{s k}(n)\right)_{*}, \quad \mathfrak{s l e}(n)=\left(\mathrm{id}, \mathfrak{s p e} \mathfrak{e}^{s k}(n)\right)_{*} .
\end{aligned}
$$


ЗАмЕчАниЕ. Картановские продолжения $\left(\mathrm{id}, \mathfrak{o s p}^{s y}(m \mid 2 n)\right)_{*}$ и $\left(\mathrm{id}, \mathfrak{p e}^{s y}(n)\right)_{*}$ конечномерны.

Обобщение картановского продолжения, описанное в п. П.5.1, имеет два супераналога: одно ассоциировано с контактной серией $\mathfrak{k}$, другое - с контактной серией $\mathfrak{m}$.

Пусть соотношение (П.16) определяет супералгебру Ли $\mathfrak{h e i}(2 n \mid m)$ или $\mathfrak{h e i l}(W)$ на прямой сумме $(2 n \mid m)$-мерного суперпространства $W$ (с невырожденной кососимметрической билинейной формой $B)$ в $(1,0)$-мерном линейном пространстве, порожденном вектором $z$. Понятно, что $\mathfrak{k}(2 n+1 \mid m)=\left(\mathfrak{h e i}(2 n \mid m), \mathfrak{c o s p}^{s k}(m \mid 2 n)\right)_{*}$. Для заданных $\mathfrak{h e i}(2 n \mid m)$ и подалгебры $\mathfrak{g}$ из $\mathfrak{c o s p}^{s k}(m \mid 2 n)$, супералгебра $(\mathfrak{h e i}(2 n \mid m), \mathfrak{g})_{*}$ называется $k$-продолжением пары $(W, \mathfrak{g})$, где $W$ - стандартный $\operatorname{cosp}(W)=\operatorname{cosp}^{s k}(m \mid 2 n)$-модуль.

"Нечетный" аналог алгебры k ассоциирован со следуюшим "нечетным" аналогом $\mathfrak{h} \mathfrak{i}(2 n \mid m)$. Пусть $\mathfrak{a b}(n)$ или $\mathfrak{a} \mathfrak{b}(W)$ обозначает "антискобочную" супералгебру Ли. Ее пространством является $W \oplus \mathbb{C} \cdot z$, где $W-(n \mid n)$-мерное суперпространство с невырожденной кососимметрической нечетной билинейной формой $B$; скобка в $\mathfrak{a} \mathfrak{b}(n)$ определяется следуюшим образом:

Элемент $z$ нечетен и лежит в центре; $[v, w]=B(v, w) \cdot z$ при $v, w \in W$.

Понятно, что $\mathfrak{m}(n)=\left(\mathfrak{a} \mathfrak{b}(n), \mathfrak{c p e} \mathfrak{e}^{s k}(n)\right)_{*}$ и для заданных $\mathfrak{a} \mathfrak{b}(n)$ и подалгебры $\mathfrak{g}$ из $\mathfrak{c p e}^{s k}(n)$ супералгебра $(\mathfrak{a} \mathfrak{b}(n), \mathfrak{g})_{*}$ называется $m$-продолжением $(W, \mathfrak{g})$, где $W$ - стандартный $\mathfrak{c p e}^{s k}(W)=\mathfrak{c p e}^{s k}(n)$-модуль.

\section{П.6. Модули тензорных полей}

Напомним определение модулей тензорных полей над $\mathfrak{v e c t}(m \mid n)$ [57], [32]. Для других $\mathbb{Z}$-градуированных векторных супералгебр Ли определение аналогично.

Пусть $\mathfrak{g}=\operatorname{vect}(m \mid n)$ и

$$
\mathfrak{g}_{\geqslant}=\bigoplus_{i \geqslant 0} \mathfrak{g}_{i}
$$

Понятно, что $\mathfrak{e e t}_{0}(m \mid n) \cong \mathfrak{g l}(m \mid n)$. Пусть $V$-это $\mathfrak{g l}(m \mid n)$-модуль с младшим весом $\lambda$.

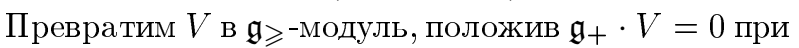

$$
\mathfrak{g}_{+}=\bigoplus_{i>0} \mathfrak{g}_{i}
$$

Представим $\mathfrak{g}$ векторными полями на $(m \mid n)$-мерном линейном супермногообразии $\mathcal{C}^{m} \mid n$ с системой координат $x=(u, \xi)$. По теореме Пуанкаре-Биркгофа-Витта суперпространство $T(V)=\operatorname{Hom}_{U(\mathfrak{g} \geqslant)}(U(\mathfrak{g}), V)$ изоморфно $\mathbb{C}[[x]] \otimes V$. Его элементы естественно интерпретировать как формальные тензорные поля типа $V$. Если $\lambda=(a, \ldots, a)$, то мы пишем просто $T(\vec{a})$ вместо $T(\lambda)$.

ПримеРЫ. 1. $\mathfrak{v e c t}(m \mid n)$, как $\mathfrak{v e c t}(m \mid n)$ - и $\mathfrak{s v e c t}(m \mid n)$-модули, - это $T$ (id).

2. $T(\overrightarrow{0})$ есть суперпространство функций.

3. $\operatorname{Vol}(m \mid n)=T(1, \ldots, 1 ;-1, \ldots,-1)$, где точка с запятой отделяет первые $m$ "четных" координат веса относительно матричных единиц $E_{i i}$ из $\mathfrak{g l}(m \mid n)$ от "нечетных" координат, есть суперпространство плотностей или форм объема. Генератор модуля 
$\operatorname{Vol}(m \mid n)$, над алгеброй функций соответствующий упорядоченному набору координат $x$, обозначается $\operatorname{vol}(x)$.

4. Пространство $\lambda$-плотностей есть $\operatorname{Vol}^{\lambda}(m \mid n)=T(\lambda, \ldots, \lambda ;-\lambda, \ldots,-\lambda)$. Его генератор обозначается $\operatorname{vol}^{\lambda}(x)$. В частности, $\operatorname{Vol}^{\lambda}(m \mid 0)=T(\vec{\lambda}), \mathrm{a} \mathrm{Vol}^{\lambda}(0 \mid n)=T(-\vec{\lambda})$.

ЗАмечаниЕ. Трактовать форму объема как произведение дифференциалов $d^{m}$ и $d^{n} \xi$ нельзя, так как березиниан никогда не возникает множителем такого выражения при заменах координат. Мы можем попытаться использовать обычные обозначения дифференциалов при условии, что все дифференциалы антикоммутируют, но на $d \xi$ надо поделить. Тогда, по крайней мере, при линейном преобразовании, которое не смешивает четные координаты $u$ с нечетными $\xi$, форма объема $\operatorname{vol}(x)$, рассматриваемая как отношение $d u_{1} \ldots d u_{m} / d \xi_{1} \ldots d \xi_{n}$, умножается на березиниан преобразования.

Действительно, пусть $x=(u, \xi)$. Для обычных внешних дифференциальных форм формы $d x_{i}$ суперантикоммутируют, поэтому формы $d \xi_{i}$ коммутируют. Если, как и в метрике, использовано симметричное произведение дифференциалов, то формы $d x_{i}$ суперкоммутируют, и, следовательно, формы $d u_{i}$ коммутируют. Однако формы $\partial / \partial \xi_{i}$ антикоммутируют и ведут себя как $1 / d \xi_{i}$ при линейных преобразованиях. Тем не менее обозначение

$$
d u_{1} \ldots d u_{m} \frac{\partial}{\partial \xi_{1}} \ldots \frac{\partial}{\partial \xi_{n}}
$$

все равно неправильное, так как почти каждое преобразование $A:(u, \xi) \mapsto(v, \eta)$ преврашает (П.17) в форму вида

$$
\operatorname{ber}(A)\left(d u^{m} \frac{\partial}{\partial \xi_{1}} \ldots \frac{\partial}{\partial \xi_{n}}\right)
$$

плюс дополнительные “лишние" слагаемые. В самом деле, отношение (П.17) является вектором старшего веса из некоторого неразложимого $\mathfrak{g l}(m \mid n)$-модуля, и vol $(x)$ обозначает образ этого вектора в одномерном фактор-модуле по инвариантному подмодулю, который порожден в точности всеми “лишними” слагаемыми.

\section{П.7. Нестандартные реализации}

В табл. 2 приведены все нестандартные градуировки введенных супералгебр Ли. В частности, градуировки серии vect задают градуировки в сериях $\mathfrak{s v e c t}$, градуировки в $\mathfrak{m}$ определяют градуировки в $\mathfrak{b}_{\lambda}, \mathfrak{l}, \mathfrak{s} \mathfrak{l} \mathfrak{e}, \mathfrak{b}, \mathfrak{s b}$, а градуировки в $\mathfrak{k}$ задают градуировки в po, h. Мы рассматриваем алгебру $\mathfrak{k}(2 n+1 \mid m)$ как сохраняющую уравнение Пфафффа вида $\alpha=0$, где

$$
\alpha=d t+\sum_{i \leqslant n}\left(p_{i} d q_{i}-q_{i} d p_{i}\right)+\sum_{j \leqslant r}\left(\xi_{j} d \eta_{j}+\eta_{j} d \xi_{j}\right)+\sum_{k \geqslant m-2 r} \theta_{k} d \theta_{k}
$$

Стандартные реализации отвечают $r=0$, они помечены $(*)$. Отметим, что коразмер- 
ность $\mathcal{L}_{0}$ минимальна в стандартной реализации.

Таблица 2

\begin{tabular}{|c|c|c|}
\hline $\begin{array}{c}\text { Супер- } \\
\text { алгебра Ли }\end{array}$ & $\begin{array}{c}\text { Параметр } \\
\text { пере- } \\
\text { градуировки } \\
\end{array}$ & $\mathbb{Z}$-градуировка \\
\hline \multirow[b]{2}{*}{$\mathfrak{v e c t}(n \mid m ; r)$} & $r=0$ & $\operatorname{deg} u_{i}=\operatorname{deg} \xi_{j}=1 \forall i, j$ \\
\hline & $0 \leqslant r \leqslant m$ & $\begin{array}{c}\operatorname{deg} \xi_{j}=0 \text { при } 1 \leqslant j \leqslant r, \\
\operatorname{deg} u_{i}=\operatorname{deg} \xi_{r+s}=1 \forall i, s\end{array}$ \\
\hline \multirow{3}{*}{$\mathfrak{m}(n ; r)$} & $r=0$ & $\begin{array}{c}\operatorname{deg} \tau=2 \\
\operatorname{deg} q_{i}=\operatorname{deg} \xi_{i}=1 \forall i\end{array}$ \\
\hline & $r=n$ & $\begin{array}{c}\operatorname{deg} \tau=\operatorname{deg} q_{i}=1 \\
\operatorname{deg} \xi_{i}=0 \forall i\end{array}$ \\
\hline & $0<r<n^{-1}$ & $\begin{array}{c}\operatorname{deg} \tau=\operatorname{deg} q_{i}=2, \\
\operatorname{deg} \xi_{i}=0 \text { для } 1 \leqslant i \leqslant r, \\
\operatorname{deg} u_{r+j}=\operatorname{deg} \xi_{r+j}=1 \forall j\end{array}$ \\
\hline \multirow[b]{2}{*}{$\mathfrak{k}(2 n+1 \mid m ; r)$} & $r=0$ & $\begin{array}{c}\operatorname{deg} t=2 \\
\operatorname{deg} p_{i}=\operatorname{deg} q_{i}=\operatorname{deg} \xi_{j}=\operatorname{deg} \eta_{j}=\quad(*) \\
=\operatorname{deg} \theta_{k}=1 \forall i, j, k\end{array}$ \\
\hline & $\begin{array}{c}0<r<\left[\frac{m}{2}\right] \\
r \neq k-1 \text { при } \\
m=2 k \text { и } n=0\end{array}$ & $\begin{array}{c}\operatorname{deg} t=\operatorname{deg} \xi_{i}=2, \operatorname{deg} \eta_{i}=0 \\
\text { при } 1 \leqslant i \leqslant r \\
\operatorname{deg} p_{i}=\operatorname{deg} q_{i}=\operatorname{deg} \theta_{j}=1 \\
\text { при } j \geqslant 1 \text { и } \forall i\end{array}$ \\
\hline $\mathfrak{k}(1 \mid 2 m ; m)$ & & $\begin{array}{c}\operatorname{deg} t=\operatorname{deg} \xi_{i}=1 \\
\operatorname{deg} \eta_{i}=0 \text { при } 1 \leqslant i \leqslant m\end{array}$ \\
\hline
\end{tabular}

Причины, по которым $r$ не может принимать значения $n-1$ для $\mathfrak{m}(n)$ и значения $k-1$ для $\mathfrak{k}(1 \mid 2 k)$, несушественны для настояшей работы, но важны при решении других задач (см., например, [39]).

Благодарности. Авторы благодарны фондам NFR (Швеция) и РФФИ (грант № 99-01-00245) за финансовую поддержку; И. Баталину, П. Грозману, Ю. Кочеткову, А. Сергееву, И. Тютину и Л. Ваксману за полезные обсуждения; М. Маринову, С. Стернбергу и М. Васильеву за вопросы, стимулировавшие наши исследования; Андрею Кириллову и Биллу Эверетту за чрезвычайно тщательное и дружелюбное редактирование, которое привело к значительному улучшению текста.

\section{Список литературы}

[1] Ю. Ю. Кочетков. Деформации супералгебр Ли. Деп. ВИНИТИ, № 384-385. М.: ВИНИТИ, 1985.

[2] Д. А. Лейmec. ДАН СССР. 1977. Т. 236. С. 804-807.

[3] B. Kostant, S. Sternberg. Ann. Phys. 1987. V. 176. № 1. P. 49-113.

[4] Д. А. Лейmec. ТМФ. 1984. Т. 58. № 2. С. 229-232; D. Leites. Quantization. Supplement 3. In: Schrödinger Equation. Eds. F. Berezin, M. Shubin. Dordrecht: Kluwer, 1991. P. 483-522.

[5] B. Fedosov. J. Diff. Geom. 1994. V. 40. № 2. P. 213-238; Deformation Quantization and Index Theory. Berlin: Akademie Verlag, 1996; I. Gelfand, V. Retakh, M. Shubin. Adv. Math. 1998. V. 136. № 1. P. 104-140. 
[6] P. Deligne. Selecta Math. (U. S. ). 1995. V. 1. № 4. P. 667-697.

[7] J. Vey. Comment. Math. Helv. 1975. V. 50. P. 421-454.

[8] Deformation Theory of Algebras and Structures and Appllications. Eds. M. Hazewinkel, M. Gerstenhaber. Dordrecht: Kluwer, 1988.

[9] F. A. Berezin. Commun. Math. Phys. 1975. V. 40. Р. 153-174; Ф. А. Березин. Изв. АН CCСР. Сер. матем. 1974. Т. 38. С. 1116-1175; 1975. T. 39. № 2. С. 363-402.

[10] И. Шерешевский. ДАН СССР. 1979. Т. 245. № 5. С. 1057-1060; I. A. Shereshevskij. Lett. Math. Phys. 1981. V. 5. № 5. P. 429-435.

[11] M. Kontsevich. Deformation quantization of Poisson manifolds. q-alg/9709040.

[12] M. Bordemann. The deformation quantization of certain super-Poisson brackets and BRST cohomology. math.QA/0003218.

[13] P. Grozman, D. Leites, I. Shchepochkina. Lie superalgebras of string theories. hep-th 9702120.

[14] I. Batalin, I. Tyutin. General local solution to the cyclic Jacobi equation (unpublished).

[15] F. Bayen, M. Flato, C. Fronsdal, A. Lichnerowicz, D. Sternheimer. Ann. Phys. 1978. V. 111. № 1. P. 61-110.

[16] A. Dzhumadildaev. Z. Phys. C. 1996. V. 72. № 3. P. 509-517.

[17] Д. А. Лейтес. УМН. 1980. Т. 35. № 1. С. 3-57; Теория супермногообразий. Петрозаводск: Карельское Отделение АН СССР, 1983.

[18] Д. Б. Фукс. Когомологии бесконечномерных алгебр Ли. М.: Наука, 1984.

[19] Ю. И. Манин. Калибровочные поля и комплексная геометрия. М.: Наука, 1984.

[20] S. Cheng, V. Kac. Adv. Theor. Math. Phys. 1998. V. 2. № 5. P. 1141-1182.

[21] J. Wess, B. Zumino. Nucl. Phys. B. 1974. V. 70. P. 39-50; J. Wess. Supersymmetry-supergravity. In: Lect. Notes Phys. V. 77. Topics in Quantum Field Theory and Gauge Theories (Proc. of VIII Internat. GIFT Sem. on Theoret. Phys., Salamanca, 1977). Ed. J.A. de Azcárraga. Berlin-New York: Springer, 1978. P. 81-125; J. Wess, B. Zumino. Phys. Lett. B. 1977. V. 66. № 4. P. 361-364; J. Wess. Supersymmetry/supergravity. In: Concepts and Trends in Particle Physics (Schladming, 1986). Eds. H. Latal and H. Mitter. Berlin: Springer, 1987. P. 29-58; Introduction to supersymmetric theories. In: Frontiers in Particle Physics '83 (Dubrovnik, 1983). Eds. Dj. Šijački, N. Bilić, B. Dragović, D. Popović. Singapore: World Sci. Publishing, 1984. P. 104-131; J. Wess, J. Bagger. Supersymmetry and Supergravity. Princeton, NJ: Princeton University Press, 1983.

[22] E. Witten. Phys. Lett. B. 1978. V. 77. № 4-5. P. 394-400; Introduction to supersymmetry in particle and nuclear physics. In: Proc. of the International School on Supersymmetry Held in Mexico City (December 14-18, 1981). Eds. O. Castanos, A. Frank, L. Urrutia. New York: Plenum, 1984. P. 53-76.

[23] Л.Е. Генденштейн, И. В. Криве. УФН. 1985. Т. 146. № 4. С. 553-590.

[24] A. Raina. C. R. Acad. Sci. Paris. Sér. I Math. 1994. V. 318. № 9. P. 851-856.

[25] M. A. Shubin. Geom. Funct. Anal. 1996. V. 6. № 2. P. 370-409; Novikov inequalities for vector fields. In: The Gelfand Seminars, 1993-1995. Eds. I. M. Gelfand et al. Boston, MA: Birkhauser, 1996. P. 243-274.

[26] J. Bernstein. The Lie superalgebra osp $(1 \mid 2)$, connections over symplectic manifolds and representations of Poisson algebras. In: Seminar on Supermanifolds. Reports of Stockholm Univ. 9/1987-13. Ed. D. Leites. Stockholm: Stockholm Univ. Press, 1987. P. 1-60; A. В. Шаповалов. Serdica. 1981. V. 7. № 4. Р. 337-342; Г. С. Шмелев. Serdica. 1982. V. 8. № 4. P. 408-417; Функц. анализ и его прилож. 1983. Т. 17. № 1. С. 91-92.

[27] Г. С. Шмелев. Функц. анализ и его прилож. 1983. Т. 17. № 4. С. 94-95; № 1. С. 91-92; Serdica. 1982. V. 8. № 4. Р. 408-417 (1983); Матем. сб. 1983. Т. 120. № 3. С. 528-539; C. R. Acad. Bulg. Sci. 1982. V. 35. № 3. P. 287-290.

[28] Ю. Ю. Кочетков. Индуцированные неприводимые представления супералгебр Лейтеса. В сб.: Вопросы теории групп и гомологической алгебры. Ред. А. Онищик. Ярославль: Ярославский гос. университет, 1983. С. 120-123; Вопр. теории групп и гомологич. алгебры. 1989. Т. 9. C. $142-148$.

[29] Ф. А. Березин. УМН. 1969. Т. 24. № 4 (148). С. 65-88. 
[30] J. Gomis, J. Paris, S. Samuel. Phys. Rep. 1995. V. 259. P. 1-191.

[31] I. Batalin, I. Tyutin. Generalized field-antifield formalism. In: Topics in Statistical and Theoretical Physics. F. A. Berezin memorial volume. Transl. of AMS. Ser. 2. V. 177. Eds. R. Dobrushin et. al. Providence, RI: AMS, 1996. P. 23-43.

[32] Д. А. Лейтес. Супералгебры Ли. В сб.: Современные проблемы математики. Новейшие достижения. Т. 25. Ред. Р. В. Гамкрелидзе. М.: ВИНИТИ, 1984. С. 3-49.

[33] Ю. Ю. Кочетков. УМН. 1989. Т. 44. № 5. С. 167-168.

[34] Yu. Kotchetkoff. C. R. Acad. Sci. Paris. Ser. I. 1984. V. 299. № 14. P. 643-645.

[35] Ю. Ю. Кочетков. Матем. заметки. 1998. Т. 63. № 3. С. 391-401; N. van den Hijligenberg, Y. Kotchetkov, G. Post. J. Algebra Comput. 1993. V. 3. № 1. P. 57-77.

[36] N. W. van den Hijligenberg, Yu. Yu. Kotchetkov. J. Math. Phys. 1996. V. 37. № 11. P. $5858-5868$.

[37] N. van den Hijligenberg, Y. Kotchetkov, G. Post. Math. Comput. 1995. V. 64. № 211. Р. 1215-1226; Ю. Ю. Кочетков. Функц. анализ и его прилож. 1994. Т. 28. № 3. С. 77-79.

[38] И. М. Щепочкина. Функц. анализ и его прилож. 1999. Т. 33. № 3. С. 59-72; hep-th/9702120; I. Shchepochkina. Represent. Theory (electr. jour. ). 1999. V. 3. P. 373-415; I. Shchepochkina, G. Post. Internat. J. Algebra Comput. 1998. V. 8. № 4. P. 479-495; physics/9703022.

[39] D. Leites, I. Shchepochkina. Classification of simple Lie superalgebras of vector fields. to appear.

[40] Д. Алексеевский, Д. Лейтес, И. Щепочкина. С. R. Acad. Bulg. Sci. 1980. V. 34. № 9. P. $1187-1190$.

[41] V. Kac. Adv. Math. 1977. V. 26. P. 8-96.

[42] В. Серганова. Функц. анализ и его прилож. 1983. Т. 17. № 3. С. 46-54; 1985. Т. 19. № 3. C. 75-76; Outer automorphisms and real forms of Kac-Moody superalgebras. In: Group Theoretical Methods in Physics (Zvenigorod, 1982). V.1-3. Eds. M.I. Markov et al. Chur: Harwood Academic Publ., 1985. P. 639-642.

[43] Г. С. Нмелев. С. R. Acad. Bulgar. Sci. 1983. V. 36. № 5. P. 569-570.

[44] A. Perelomov. Integrable Systems of Classical Mechanics and Lie Algebras V.I. Basel: Birkhäuser, 1990.

[45] C. Duval, P. Lecomte, V. Ovsienko. Ann. Inst. Fourier (Grenoble). 1999. V. 49. № 6. P. 1999-2029; math.DG/9902032.

[46] P. B. A. Lecomte, V. Yu. Ovsienko. Projectively equivariant symbol calculus. math.DG/ 9809061; Lett. Math. Phys. 1999. V. 49. № 3. P. 173-196.

[47] D. Leites, E. Poletaeva. Math. Scand. 1997. V. 81. № 1. P. 5-19.

[48] P. Grozman, D. Leites, E. Poletaeva. Defining relations for simple Lie superalgebras of polynomial vector fields. In: Supersymmetries and Quantum Symmetries. Eds. E. Ivanov et. al (SQS'99, 27-31 July, 1999). Dubna: JINR, 2000. P. 387-396.

[49] R. Floreanini, D. Leites, L. Vinet. On the defining relations of quantum Poisson superalgebras (to appear).

[50] D. Leites, A. Shapovalov. J. Nonlinear Math. Phys. 2000. V. 7. № 2. P. 120-125.

[51] П. Я. Грозман. Функц. анализ и его прилож. 1980. Т. 14. № 2. С. 58-59.

[52] V. Guillemin, Sh. Sternberg. Symplectic Techniques in Physics. Cambridge: Cambridge University Press, 1990.

[53] D. B. Fuchs, D. Leites. C. R. Acad. Bulg. Sci. 1984. V. 37. № 12. P. 1595-1596.

[54] A. Sergeev. An analog of the classical invariant theory for Lie superalgebras. math.RT/9810113.

[55] Quantum Fields and Strings: A Course for Mathematicians. V.1, 2. Material from the Special Year on Quantum Field Theory Held at the Institute for Advanced Study, 1996-1997. Eds. P. Deligne et al. Providence, RI - Princeton, NJ: AMS-IAS, 1999.

[56] A. Sergeev. Irreducible representations of solvable Lie superalgebras. math.RT/9810109; Represent. Theory (electr. jour. ). 1999. V. 3. P. 435-443.

[57] J. Bernstein, D. Leites. Sel. Math. Sov. 1981. V. 1. № 2. P. 143-160.

Поступила в редакцию $8 . \mathrm{IV} .2000$ г., после доработки 2.Х.2000 г.

2 Теоретическая и математическая физика, т. 126, № 3, 2001 г. 